e) Die experimentelle Anordnung kann von der in ${ }^{1}$ beschriebenen Form sein.

\section{$\S 10$. Fortsetzung der Untersuchungen}

Zur Klärung der in $\S 7$ behandelten Punkte werden numerische Berechnungen durchgeführt. Dabei wird analog $\mathrm{zu}$ früheren Betrachtungen ${ }^{1}$ vorgegangen. Repräsentative Verhältnisse der ungestörten irdischen Tages-Ionosphäre werden zugrunde gelegt und dafür Fehlerfortpflanzung, Dämpfung, Grenzen der WKB-Theorie, weitere Einflüsse von Reflexion und Kopplung sowie Strahlversetzung untersucht. Über die Ergebnisse dieser Arbeiten wird getrennt berichtet.

\section{Anerkennung}

Herrn Professor Dr. W. Dieminger, Direktor des Max-Planck-Institutes für Aeronomie, Lindau (Harz), danke ich für die Ermöglichung dieser und der vorangegangenen Untersuchungen, für stetes Interesse und beständige Förderung sowie für richtunggebende Diskussionen.

\title{
Zur Begrenzung der radialen Ausdehnung des Lichtbogenstromes durch ein axiales Magnetfeld
}

\author{
I. Problemstellung, Bogenmodell und typische Resultate \\ O. KLÜBER \\ Institut für Plasmaphysik, 8046 Garching b. München \\ (Z. Naturforsch. 25 a, 1583-1600 [1970]; eingegangen am 16. Juli 1970)
}

\begin{abstract}
In an arc without external magnetic field the current carrying region is identical with the conducting plasma column. This is no longer generally true if the arc is in an axial magnetic field and if the electrode radius is much smaller than the plasma radius. Radial current components then produce a rotational motion of the plasma and an azimuthal Hall current, and hence electromotive forces which try to suppress the current perpendicular to the magnetic field. In a plasma with finite viscosity the rotation is determined by the Navier-Stokes equation, which is solved here for a homogeneous plasma simultaneously with generalized Ohm's law. The results show that the plasma rotation is always an essential, and often the dominant, mechanism for guiding the arc current parallel to the magnetic field lines.
\end{abstract}

\section{Problemstellung}

In einer vorangegangenen Arbeit ${ }^{1}$ wurde die Stromdichteverteilung eines Lichtbogens im Magnetfeld einer zylindrischen Spule experimentell untersucht. Es handelte sich dort um eine Anordnung, bei der der magnetische Fluß durch beide Elektrodenquerschnitte der gleiche war; daher konnte der Bogenstrom von der Anode zur Kathode fließen, ohne die Feldlinien des Spulenfeldes zu schneiden. Es zeigte sich, daß der Stromquerschnitt überall praktisch mit dem Querschnitt der durch die Wahl der Elektrodenradien ausgezeichneten magnetischen Flußröhre zusammenfällt, obwohl die leitfähige Plasmasäule über einen beträchtlichen Teil der Bogenlänge einen erbeblich größeren Querschnitt als diese ausgezeichnete Flußröhre hat. Als wesentliche Ursache für den „Einschluß“ des Bogen-

Sonderdruckanforderungen an Dr. O. KLÜBER, Institut für Plasmaphysik, $D$-8046 Garching bei München. stroms wurde die Rotationsbewegung des Plasmas erkannt. Sie führt zu einer Potentialverteilung derart, daß das zum Spulenfeld senkrechte elektrische Feld durch $\boldsymbol{v} \times \boldsymbol{B}$ kompensiert wird, und daß das zu $\boldsymbol{B}$ parallele elektrische Feld und damit auch die Stromdichte außerhalb der ausgezeichneten magnetischen Flußröhre verschwindet.

Ganz wesentlich für das Funktionieren dieses Mechanismus ist, ob die Rotation des Plasmas durch die innere Reibung merklich beeinflußt wird oder nicht. Das zeigt sich, wenn man die Einstellung der stationären Stromdichte- und Potentialverteilung eines Lichtbogens anhand der Gln.

$$
\begin{aligned}
& \operatorname{div} \boldsymbol{j}=0, \\
& \operatorname{rot} \boldsymbol{E}=0, \\
& \frac{1}{\sigma} \boldsymbol{j}=\boldsymbol{E}+\boldsymbol{v} \times \boldsymbol{B}-\frac{1}{e n_{\mathrm{e}}} \boldsymbol{j} \times \boldsymbol{B}+\frac{1}{e n_{\mathrm{e}}} \nabla p_{\mathrm{e}},
\end{aligned}
$$




$$
\varrho \frac{\mathrm{d} \boldsymbol{v}}{\mathrm{d} t}+\nabla \boldsymbol{p}+D(\mu \boldsymbol{v})=\boldsymbol{j} \times \boldsymbol{B}
$$

diskutiert. In Gl. (4) (Navier-Stokes-Gl.) ist die innere Reibung des Plasmas durch den Term $D(\mu \boldsymbol{v})$ berücksichtigt; dabei ist $\mu$ die Viskosität des Plasmas und $D(\mu \boldsymbol{v})$ ein Differentialausdruck zweiter Ordnung in den Ortskoordinaten von $\boldsymbol{v}$. Alle anderen Größen haben die bekannte Bedeutung; es wird das VAcs-System benutzt.

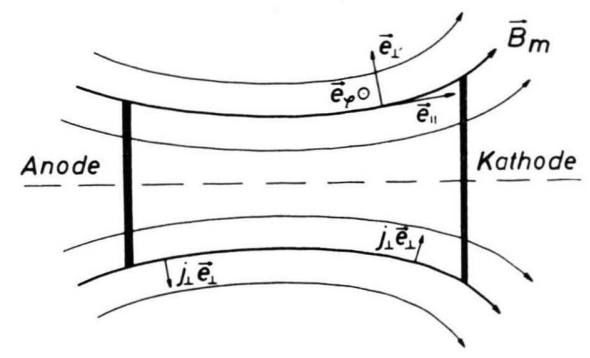

Abb. 1. Schematische Darstellung eines Lichtbogens mit überlagertem Magnetfeld.

Die zunächst betrachtete Geometrie des Bogens ist in Abb. 1 schematisch dargestellt. Der magnetische Fluß durch beide Elektrodenquerschnitte ist der gleiche, wie es auch im anfangs erwähnten Experiment der Fall war. Es ist für die Einstellung der Stromdichteverteilung prinzipiell belanglos, ob die Feldlinien des äußeren Magnetfeldes gekrümmt sind oder nicht. Dieses Magnetfeld ist als meridionales Magnetfeld mit $\boldsymbol{B}_{\mathrm{m}}$ bezeichnet; das gesamte Magnetfeld ist $\boldsymbol{B}=\boldsymbol{B}_{\mathrm{m}}+B_{\varphi} \boldsymbol{e}_{\varphi}$. Es ist zweckmäßig, ein der Geometrie der $\boldsymbol{B}_{\mathrm{m}}$-Linien angepaßtes Koordinatensystem mit den Einheitsvektoren $\boldsymbol{e}_{\|}=\boldsymbol{B}_{\mathrm{m}} / B_{\mathrm{m}}, \boldsymbol{e}_{\varphi}$ und $\boldsymbol{e}_{\perp}=\boldsymbol{e}_{\varphi} \times \boldsymbol{e}_{\|}$zu verwenden. Dann ist per def. $B_{\perp}=0$. Um nun zu zeigen, welche stationäre Stromdichteverteilung sich in einem solchen Lichtbogen einstellt, soll ein Ausgangszustand mit $j_{\perp}=0$ betrachtet werden, d.h. der Bogenstrom soll anfangs genau parallel zu den $\boldsymbol{B}_{\mathrm{m}}$-Linien fließen. Er soll ferner die Umgebung der stromführenden Bogensäule so stark aufheizen, daß auch außerhalb der ausgezeichneten magnetischen Flußröhre leitfähiges Plasma vorhanden ist. Da anfangs auch dort das an die Elektroden angelegte elektrische Feld herrscht, hat der Strom die Tendenz, sich über die durch den Elektrodenquerschnitt bestimmte Flußröhre hinaus auszudehnen. Es wird also an der Anode eine Stromdichtekomponente $j_{\perp}>0$ und entsprechend an der Kathode eine Komponente $j_{\perp}<0$ auftreten. Dieser Ausdeh- nung des Bogenstromes wirken aber elektromotorische Kräfte entgegen: Nach Gl. (4) versetzt die azimutale Kraftdichte $-j_{\perp} B_{\mathrm{m}} \boldsymbol{e}_{\varphi}$ das Plasma in Rotation, deren Drehsinn vor beiden Elektroden entgegengesetzt ist, und zwar gerade so, da $\beta$ die EMK $v_{\varphi} B_{\mathrm{m}} \boldsymbol{e}_{\perp}$ nach Gl. (3) - und entsprechend der Lenz'schen Regel - der Stromdichte $j_{\perp}$ entgegengerichtet ist. Weiterhin ist nach Gl. (3)

$$
j_{\varphi}=\frac{\sigma}{e n_{\mathrm{e}}} j_{\perp} B_{\mathrm{m}}-\sigma v_{\perp} B_{\mathrm{m}}=j_{\varphi}^{(\mathrm{H})}-\sigma v_{\perp} B_{\mathrm{m}} .
$$

Die durch diese Gl. gegebene azimutale HallStromdichte $j_{\varphi}^{(\mathrm{H})}$ führt - wieder über den HallTerm - zur Hall-EMK $-\sigma B_{m}^{2} j_{\perp} \boldsymbol{e}_{\perp} / e^{2} n_{e}^{2}$, die ebenfalls der Stromdichte $j_{\perp} \boldsymbol{e}_{\perp}$ entgegenwirkt.

Der stationäre Endzustand, der sich unter der Wirkung dieser beiden elektromotorischen Kräfte einstellt, läßt sich sofort angeben, wenn man die innere Reibung des Plasmas außer acht läßt, d.h. in Gl. (4) $\mu=0$ setzt und dadurch zur Eulerschen Gl. übergeht. Dann besagt Gl. (4) folgendes: Da der azimutalen Kraftdichte $-j_{\perp} B_{\mathrm{m}} \boldsymbol{e}_{\varphi}$ wegen der vorausgesetzten Rotationssymmetrie nicht durch einen Druckgradienten das Gleichgewicht gehalten werden kann, ist $(\mathrm{d} \boldsymbol{v} / \mathrm{dt}) \neq 0$ für $j_{\perp} \neq 0$. Wegen

$$
\frac{\mathrm{d} \boldsymbol{v}}{\mathrm{d} t}=\frac{\partial \boldsymbol{v}}{\partial t}+(\boldsymbol{v} \nabla) \boldsymbol{v}
$$

folgt daraus noch nicht unmittelbar $\partial v_{\varphi} / \partial t \neq 0$. Zieht man jedoch noch den Energiesatz für die kinetische Energie heran, so ergibt sich, wie an anderer Stelle ${ }^{1}$ gezeigt wurde, daß die Arbeit $-j_{\perp} B_{\mathrm{m}} v_{\varphi}$ nur zur Erhöhung der Rotationsenergie führen kann. Deswegen nimmt in einem als reibungsfrei gedachten Plasma die Rotationsgeschwindigkeit zu, solange $j_{\perp} \neq 0$ ist. Das stationäre Gleichgewicht ist also dann erreicht, wenn $v_{\varphi}$ so weit angewachsen ist, daß die EMK $v_{\varphi} B_{\mathrm{m}} \boldsymbol{e}_{\perp}$ die Stromdichte $j_{\perp}$ zum Erliegen gebracht hat. Mit $j_{\perp}$ ist dann nach Gl. (3) auch der azimutale Hall-Strom verschwunden, der somit im reibungsfreien Plasma nur während des Einstellvorganges eine Rolle spielt.

Für ein reibungsfreies Plasma gilt demnach im stationären Fall $j_{\perp}=0$. Aus Gl. (3) erhält man dann

$$
\begin{gathered}
\frac{1}{\sigma} j_{\|}=E_{\|}+v_{\perp} B_{\varphi}+\frac{1}{e n_{\mathrm{e}}} \nabla_{\|} p_{\mathrm{e}}, \\
0=E_{\perp}+v_{\varphi} B_{\mathrm{m}}-v_{\|} B_{\varphi}+\frac{1}{e n_{\mathrm{e}}} \nabla_{\perp} p_{\mathrm{e}} .
\end{gathered}
$$

Da nun kein Strom die durch die Wahl der Elektrodenradien ausgezeichnete magnetische Fluß- 
röhre verläßt, muß $j_{\|}$außerhalb dieser Flußröhre verschwinden. $E_{\|} \mathrm{mu}$ also dort die elektromotorischen Kräfte $v_{\perp} B_{\varphi}$ und $\nabla_{\|} p_{\mathrm{e}} / e n_{\mathrm{e}}$ kompensieren. Das dazu erforderliche elektrische Feld ist in den meisten Fällen klein gegen $E_{\|} \approx j_{\|} / \sigma$ innerhalb der ausgezeichneten Flußröhre. Gleichwohl ist $\operatorname{rot} \boldsymbol{E}=\mathbf{0}$ erfüllt, da $E_{\perp} \approx-v_{\varphi} B_{\mathrm{m}}$ sich mit $v_{\varphi}$ über die Bogenlänge ändert; $v_{\varphi}$ hat ja vor den beiden Elektroden entgegengesetztes Vorzeichen. Bildet man $\oint \boldsymbol{E} \mathrm{d} \boldsymbol{s}$ für einen Integrationsweg, der teils innerhalb, teils außerhalb der ausgezeichneten Flußröhre verläuft, so kompensieren die Beiträge von $E_{\perp}$ gerade den Beitrag, den $E_{\|}$innerhalb der den Strom begrenzenden Flußröhre liefert.

In einem realen Plasma mit endlicher innerer Reibung sind demgegenüber stationäre Zustände mit $j_{\perp} \neq 0$ zugelassen. Die von dem zu $\boldsymbol{B}_{\mathrm{m}}$ senkrechten Anteil des Stromes am Plasma geleistete Arbeit $-\int j_{\perp} B_{\mathrm{m}} v_{\varphi} \mathrm{d} V$ (sie ist positiv, weil $v_{\varphi}$ das Vorzeichen von $-j_{\perp}$ hat) kann dann durch die innere Reibung in Wärme umgewandelt werden; sie muß deshalb nicht zur Erhöhung der Rotationsenergie führen. Eine stationäre Stromdichteverteilung mit $j_{\perp} \neq 0$ wird dann natürlich auch durch den Hall-Strom mitbestimmt. Ob ein solcher stationärer Zustand eines reibenden Plasmas sich von dem eines reibungsfreien Plasmas wesentlich unterscheidet, hängt dabei nicht nur von den Plasmaeigenschaften, sondern auch von der Geometrie der Anordnung ab. Der Einfluß der inneren Reibung des Plasmas muß sich offenbar dann besonders drastisch bemerkbar machen, wenn die Elektroden so angeordnet sind, daß jede $\boldsymbol{j}_{\mathrm{m}}$-Linie die $\boldsymbol{B}_{\mathrm{m}}$-Linien schneiden muß. In einem reibungsfreien Plasma würde dann im Endzustand kein Strom mehr flieBen; das zwischen den Elektroden angelegte elektrische Feld würde vielmehr völlig durch $\boldsymbol{v} \times \boldsymbol{B} \mathrm{kom}$ pensiert werden. In einem realen Plasma fließt dagegen ein Strom quer zu den magnetischen Feldlinien.

Das Ziel dieser Arbeit ist, quantitativ zu untersuchen, wie sich die stationäre Stromdichteverteilung eines Lichtbogens in einem äußeren Magnetfeld in verschiedenen Geometrien bei Berücksichtigung der endlichen inneren Reibung einstellt. Dazu hat man die Navier-Stokes'sche Gleichung (4) simultan mit den Gln. (1) bis (3) zu lösen. Wegen der Kompliziertheit des Problems ist das nur unter vereinfachenden Annahmen möglich. Es wird deshalb ein Modellbogen in einfacher Geometrie betrachtet.

\section{Das Bogenmodell}

Den Untersuchungen wird ein Bogenmodell zugrundegelegt, das durch folgende Eigenschaften gekennzeichnet ist:

(1) Das äußere Magnetfeld sei homogen; es habe nur eine $z$-Komponente. Dann fallen die im vorigen Abschnitt eingeführten, der Feldgeometrie angepaßten Koordinaten mit den Zylinderkoordinaten zusammen, d.h. es ist $j_{\perp}=j_{r}, j_{\|}=j_{z}$. Das Eigenmagnetfeld $\boldsymbol{B}_{\varphi} \boldsymbol{e}_{\varphi}$ des Bogenstromes wird vernachlässigt: $\boldsymbol{B}=(0,0, B)$.

(2) Das Plasma sei in radialer Richtung unendlich ausgedehnt und werde in axialer Richtung durch zwei ebene Wände begrenzt, die bei $-z_{0}$ und $+z_{0}$ liegen. Auf diesen Wänden befinden sich die Elektroden, die Anode bei $-z_{0}$, die Kathode bei $+z_{0}$, so daß $j_{z}>0$ gilt. $r_{\mathrm{K}}$ und $r_{\mathrm{A}}$ seien die Innenradien, $R_{\mathrm{K}}$ und $R_{\mathrm{A}}$ die Außenradien der Elektroden (vgl. Abb. 2).

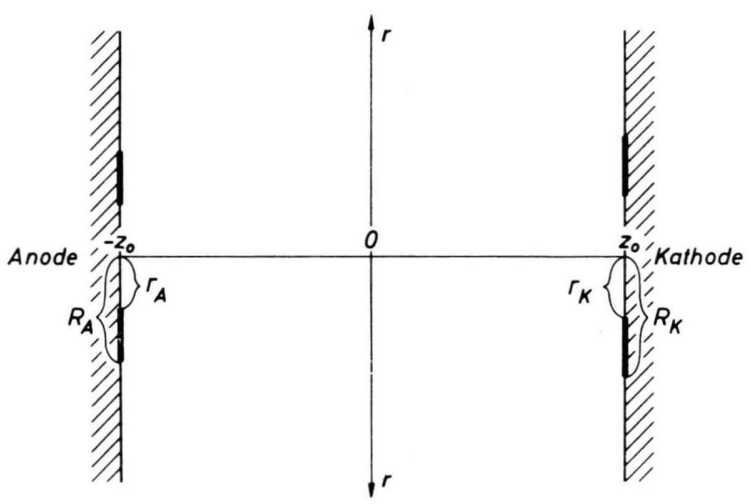

Abb. 2. Geometrie des Bogenmodells.

(3) Das Plasma sei homogen, d.h. Dichte und Temperatur seien keine Ortsfunktionen. Dann sind auch Leitfähigkeit und Viskosität ortsunabhängig; ferner verschwindet dann $\nabla p_{\mathrm{e}}$. Weiter wird angenommen, daß in der $r-z$-Ebene keine Plasmabewegung stattfindet, so daß $\boldsymbol{v}=\left(0, v_{\varphi}, 0\right)$ gilt.

Unter diesen Voraussetzungen erhält man für Zylinderkoordinaten aus Gl. (3) die Komponentengleichungen

$$
\begin{aligned}
& \frac{1}{\sigma} j_{r}=E_{r}+v_{\varphi} B-\frac{1}{e n_{\mathrm{e}}} j_{\varphi} B, \\
& \frac{1}{\sigma} j_{\varphi}=\frac{1}{e n_{\mathrm{e}}} j_{r} B, \\
& \frac{1}{\sigma} j_{z}=E_{z} .
\end{aligned}
$$


Setzt man Gl. $(3 \varphi)$ die den azimutalen Hall-Strom angibt, in Gl. (3r) ein, so erhält man mit

$$
\sigma=\frac{e^{2} n_{\mathrm{e}} \tau_{\mathrm{e}}}{m_{\mathrm{e}}},
$$

wo $\tau_{\mathrm{e}}$ die reziproke Stoßfrequenz der Elektronen ist,

$$
\frac{1}{\sigma}\left(1+\omega_{\mathrm{e}}^{2} \tau_{\mathrm{e}^{2}}\right) j_{r}=E_{r}+v_{\varphi} B .
$$

$\omega_{\mathrm{e}}$ ist dabei die Gyrofrequenz der Elektronen.

Berücksichtigt man $\operatorname{rot} \boldsymbol{E}=0$ (Gl. (2)) in der üblichen Weise durch

$$
\boldsymbol{E}=-\operatorname{grad} V
$$

und führt noch

$$
\sigma^{\prime}=\frac{\sigma}{1+\omega_{\mathrm{e}}^{2}} \tau_{\mathrm{e}^{2}}
$$

als ,Leitfähigkeit senkrecht zu B“" ein, so ergibt sich

$$
\begin{aligned}
& j_{r}=-\sigma^{\prime} \frac{\partial V}{\partial r}+\sigma^{\prime} v_{\varphi} B, \\
& j_{z}=-\sigma \frac{\partial V}{\partial z}
\end{aligned}
$$

als Kombination der Gln. (2) und (3r) bis (3z). Einsetzen in Gl. (1) liefert dann nach Division durch $\sigma^{\prime}$ die Gl.

$$
\frac{1}{r} \frac{\partial}{\partial r}\left(r \frac{\partial V}{\partial r}\right)+\frac{\sigma}{\sigma^{\prime}} \frac{\partial^{2} V}{\partial z^{2}}=\frac{1}{r} \frac{\partial}{\partial r}\left(r v_{\varphi}\right) B .
$$

Vergleicht man diese Gl. mit der Potentialgleichung

$$
\Delta V \equiv \frac{1}{r} \frac{\partial}{\partial r}\left(r \frac{\partial V}{\partial r}\right)+\frac{\partial^{2} V}{\partial z^{2}}=\varepsilon_{0} \varrho_{\mathrm{e}},
$$

so sieht man, daß die elektrische Raumladungs- dichte $\varrho_{\mathrm{e}}$ durch

$$
\varepsilon_{0} \varrho_{\mathrm{e}}=\omega_{\mathrm{e}}^{2} \tau_{\mathrm{e}}^{2} \frac{\partial^{2} V}{\partial z^{2}}-\frac{1}{r} \frac{\partial}{\partial r}\left(r v_{\varphi}\right) B
$$

gegeben ist. Im Fall $B=0$ verschwindet die Ladungsdichte, so daß $\boldsymbol{E}$ gleich dem an die Elektroden angelegten elektrischen Feld ist. Im Fall $B \neq 0$ wird dieses Feld durch Plasmarotation und Hall-Strom verzerrt.

Spezielle Lösungen der Gl. (5), nämlich solche für $v_{\varphi}=0$, wurden von RAEDER und $\mathrm{WIRTZ}^{2}$ angegeben. Nun trifft $v_{\varphi}=0$ sicher für $B=0 \mathrm{zu}$, da es dann keine Kräfte gibt, die zu einer Rotation des Plasmas führen könnten. Für $\mathrm{B} \neq 0$ stellt die Annahme $v_{\varphi}=0$ jedoch eine zusätzliche Annahme über die Eigenschaften des Plasmas dar. Sucht man nach einem Modell, das $v_{\varphi}=0$ liefert, so könnte man an ein Plasma denken, in dem die Ionenmasse als unendlich angenommen wird. Dann wird der elektrische Strom ausschließlich (und nicht nur überwiegend wie im Plasma mit Ionen endlicher Masse) von den Elektronen getragen und vom Magnetfeld nur über den Hall-Term beeinflußt. Im Hinblick auf die Stromverteilung verhält sich ein solches Plasma also wie ein festes Metall. Das Modell des homogenen, reibend rotierenden Plasmas entspricht demgegenüber einem flüssigen Metall. Der Vergleich der Lösungen für $v_{\varphi}=0$ und $v_{\varphi} \neq 0$ liefert einen Einblick in die Rolle der Ionenbewegung für die Einstellung der Stromdichteverteilung. Er läßt sich am einfachsten für punktförmige Elek troden durchführen. Befinden sich diese bei $\left(0,-z_{0}\right)$ und $\left(0, z_{0}\right)$, so lautet die Lösung der Gl. (5) für $v_{\varphi}=0$

$$
\left.\left.V^{(0)}(r, z)=\frac{I}{4 \pi \sqrt{\sigma \sigma^{\prime}}}\left\{\mid r^{2}+\frac{\sigma^{\prime}}{\sigma}\left(z+z_{0}\right)^{2}\right]^{-1 / 2}-\mid r^{2}+\frac{\sigma^{\prime}}{\sigma}\left(z-z_{0}\right)^{2}\right]^{-1 / 2}\right\},
$$

wobei der Index (0) auf die Annahme $v_{\varphi}=0$ hinweist und zur Unterscheidung zu der Lösung für $v_{\varphi} \neq 0$ dient. Aus Gl. (6) läßt sich sofort die Stromdichte $j_{z}^{(0)}=-\sigma \partial V^{(0)} / \partial z$ bestimmen. Sie ist, wie schon anschaulich klar ist, bei $z=0$ am stärksten verbreitert. Dort gilt

$$
-\left.\sigma \frac{\partial V^{(0)}}{\partial z}\right|_{z=0}=\frac{I}{2 \pi} \frac{\sqrt{\left(\sigma^{\prime} / \sigma\right)} z_{0}}{\left(r^{2}+\left(\sigma^{\prime} / \sigma\right) z_{0}^{2}\right)^{3 / 2}} .
$$

Die Breite der Stromdichteverteilung $j_{z}^{(0)}$ ist somit durch

$$
z_{0}^{\prime}=\sqrt{\frac{\sigma^{\prime}}{\sigma}} z_{0}=\frac{1}{\sqrt{1+\omega_{\mathrm{e}}{ }^{2} \tau_{\mathrm{e}}{ }^{2}}} z_{0}
$$

2 J. RAeder u. S. Wirtz, Z. Naturforsch. 24a, 1433 [1969]. charakterisiert. Mit der Verteilung (7) sollen spätere Resultate verglichen werden.

Für rotierendes Plasma hat man Gl. (5) simultan mit der $\varphi$-Komponente der Navier-Stokes-Gleichung (4) zu lösen. Um diese Gleichung zu erhalten, muß man den verallgemeinerten Drucktensor betrachten, in dessen Komponenten Reibungsterme der Form $\mu_{\alpha} \partial v_{i} / \partial x_{k}$ auftreten. Die Viskositätskoeffizienten $\mu_{\alpha}$ hängen dabei von den Plasmaeigenschaften und den Richtungen der Bewegung und des Impulstransports relativ zum Magnetfeld ab. Da die Viskosität des Elektronengases stets klein gegen die des Ionengases ist, ist die Viskosität 
des Plasmas praktisch mit der der Ionenkomponente identisch. Solange das Produkt aus der Ionengyrofrequenz $\omega_{\mathrm{i}}$ und der freien Flugzeit $\tau_{\mathrm{i}}$ der Ionen klein gegen 1 ist, ist das Plasma praktisch isotrop. Die Viskosität ist dann

$$
\mu=n_{\mathrm{i}} \tau_{\mathrm{i}} k T .
$$

Für Bewegungen senkrecht zu $\boldsymbol{B}$ ist die Viskosität, wenn sich die Geschwindigkeit parallel zu B ändert, nach BRAGINSKY ${ }^{3}$ durch

$$
\begin{aligned}
\mu^{\prime} & =\mu P^{\prime}\left(\omega_{\mathrm{i}} \tau_{\mathrm{i}}\right), \\
P^{\prime}\left(\omega_{\mathrm{i}} \tau_{\mathrm{i}}\right) & =\left\{\begin{array}{l}
1 \text { für } \omega_{\mathrm{i}}^{2} \tau_{\mathrm{i}}^{2} \ll 1, \\
\frac{6}{5 \omega_{\mathrm{i}}{ }^{2} \tau_{\mathrm{i}}{ }^{2}} \text { für } \omega_{\mathrm{i}}^{2} \tau_{\mathrm{i}}^{2} \gg 1
\end{array}\right.
\end{aligned}
$$

gegeben. (Die explizite Form für $P^{\prime}$ findet sich ebenfalls bei Braginsky, soll aber hier nicht angegeben werden.) Für Bewegungen senkrecht zu $\boldsymbol{B}$ bei denen sich die Geschwindigkeit auch senkrecht zu $\boldsymbol{B}$ ändert, ist, ebenfalls nach Braginsky, die Viskosität

$$
\mu^{\prime \prime}=\mu P^{\prime \prime}\left(\omega_{\mathrm{i}} \tau_{\mathrm{i}}\right), \quad P^{\prime \prime}\left(\omega_{\mathrm{i}} \tau_{\mathrm{i}}\right)=P^{\prime}\left(2 \omega_{\mathrm{i}} \tau_{\mathrm{i}}\right) .
$$

Für $\omega_{\mathrm{i}}^{2} \tau_{\mathrm{i}}^{2} \gg 1$ ist somit $\mu^{\prime \prime}=\mu^{\prime} / 4$. Unter Benutzung der beiden Viskositäten $\mu^{\prime}$ und $\mu^{\prime \prime}$ erhält man in Zylinderkoordinaten für die $\varphi$-Komponente der Navier-Stokes-Gleichung den Ausdruck 4

$$
-\mu^{\prime \prime} \frac{\partial}{\partial r}\left[\frac{1}{r} \frac{\partial}{\partial r}\left(r v_{\varphi}\right)\right]-\mu^{\prime} \frac{\partial^{2} v_{\varphi}}{\partial z^{2}}=-j_{r} B
$$

Setzt man Gl. $\left(3 r^{\prime}\right)$ für $j_{\mathrm{r}}$ ein und dividiert durch $\mu^{\prime \prime}$, so folgt

$$
\frac{\partial}{\partial r}\left[\frac{1}{r} \frac{\partial}{\partial r}\left(r v_{\varphi}\right)\right]+\frac{\mu^{\prime}}{\mu^{\prime \prime}} \frac{\partial^{2} v_{\varphi}}{\partial z^{2}}-\frac{\sigma^{\prime} B^{2}}{\mu^{\prime \prime}} v_{\varphi}=-\frac{\sigma^{\prime} B}{\mu^{\prime \prime}} \frac{\partial V}{\partial r} .
$$

Die Gln. (5) und (8) beschreiben die stationäre Potentialverteilung $V(r, z)$ und die stationäre Rotationsbewegung $v_{\varphi}(r, z)$ in einem Plasma mit endlicher innerer Reibung. Kenntnis dieser beiden Größen gestattet dann die Bestimmung der Verteilung $\left(j_{\mathrm{r}}, 0, j_{z}\right)$ des Bogenstromes nach den Gln. $\left(3^{\prime} \mathrm{r}\right)$ und $\left(3^{\prime} \mathrm{z}\right)$. Die Randbedingungen für $V$ und $v_{\varphi}$ sind dabei die folgenden: Das Potential ist auf den Elektroden konstant und verschwindet für $r \rightarrow \infty$. Die $z$-Komponente $j_{z}=-\sigma \partial V / \partial z$ der Stromdichte verschwindet in den Ebenen $z=-z_{0}$ und $z=z_{0}$ außer auf den Elektroden selbst. Die Rotationsgeschwindigkeit verschwindet für $r=0, r \rightarrow \infty$ und $z= \pm z_{0}$, und zwar aus folgenden Gründen: Für $r=0 \mathrm{muß} v_{\varphi}=0$ gelten, weil sonst die Reibungskraftdichte, nämlich der Term $\mu^{\prime \prime} v_{\varphi} / r^{2}$, divergiert. Für $r \rightarrow \infty \mathrm{muß} v_{\varphi}$ verschwinden, weil sonst die kinetische Rotationsenergie $\varrho / 2 \int v_{\varphi}^{2} \mathrm{~d} V$ divergiert. Das Verschwinden an den Berandungen $z= \pm z_{0}$ ist $\mathrm{zu}$ fordern, wenn außer der inneren Reibung des Plasmas sinnvollerweise auch die Reibung des Plasmas an der Wand berücksichtigt wird. Diese beeinflußt die Rotationsgeschwindigkeit natürlich ganz wesentlich, denn es können auch in reibenden Medien reibungsfreie Bewegungen auftreten. Setzt man z.B. den Ansatz $v_{\psi}=$ const $r z$ in Gl. (8) ein, so erhält man

3 S. I. Braginsky in: M. A. Leontovich: Reviews of Plasma Physics, Vol. 1, Consultants Bureau, New York, [1967].

4 nach einer privaten Mitteilung von S. WIRTZ.

$$
\frac{\partial}{\partial r}\left[\frac{1}{r} \frac{\partial}{\partial r}\left(r v_{\varphi}\right)\right]=0, \frac{\partial^{2} v_{\varphi}}{\partial z^{2}}=0 ; \frac{\partial V}{\partial r}=v_{\varphi} B .
$$

Nur ist eine solche Bewegung nicht mit den Randbedingungen verträglich: $v_{\varphi}$ verschwindet nicht an den Wänden $z= \pm z_{0}$ und nicht für $r \rightarrow \infty$. (Würde man das Plasma auch in radialer Richtung durch eine zylindrische Wand begrenzen, so wäre auch dort das Verschwinden von $v_{\varphi}$ zu fordern.)

Die räumliche Verteilung der Rotationsgeschwindigkeit und damit auch die auftretende Reibungskraft hängt somit ganz wesentlich von der Geometrie des Bogens ab. Nun gibt es eine charakteristische Länge, die ein Maß für den Einfluß der inneren Reibung auf die Stromdichteverteilung darstellt. Nach Gl. (8) ist nämlich die innere Reibung überall dort vernachlässigbar, wo

$$
\frac{\partial}{\partial r}\left[\frac{1}{r} \frac{\partial}{\partial r}\left(r v_{\psi}\right)\right]+\frac{\mu^{\prime}}{\mu^{\prime \prime}} \frac{\partial^{2} v_{\varphi}}{\partial z^{2}} \ll \frac{\sigma^{\prime} B^{2}}{\mu^{\prime \prime}} v_{\varphi}=\frac{v_{\varphi}}{a^{2}}
$$

ist, weil dann nach Gl. (8) näherungsweise $v_{\varphi} B=$ $\partial V / \partial r$ gilt und das Plasma damit praktisch reibungsfrei ist. Die durch die obige Beziehung definierte Größe $a$ hat, wie man sofort sieht, die Dimension einer Länge. Setzt man nun den Differentialausdruck in dieser Beziehung gleich $v_{\varphi} / b^{2}$, so muß $b$ (wie sich durch Koordinatentransformation zeigen läßt) mit Vergrößerung der Bogenabmessungen (Elektrodenradien und -abstand) wachsen. Der Einfluß der inneren Reibung wird also gering sein, wenn $a$ hinreichend klein gegen die geometrischen Bogenparameter ist. Die charakteristische Länge $a$ 
ist aber in den praktisch interessierenden Fällen meistens sehr klein. Unter Benutzung der Definitionsgleichungen für $\sigma, \sigma^{\prime}$ und $\mu^{\prime}, \mu^{\prime \prime}$ erhält man nämlich

$$
a=\left(\frac{\mu^{\prime \prime}}{\sigma^{\prime} B^{2}}\right)^{1 / 2}=P^{\prime \prime 1 / 2}\left(1+\omega_{\mathrm{e}}^{2} \tau_{\mathrm{e}^{2}}\right)^{1 / 2}\left(\frac{n_{\mathrm{i}} \tau_{\mathrm{i}} m_{\mathrm{e}} k T}{n_{\mathrm{e}} \tau_{\mathrm{e}} e^{2} B^{2}}\right)^{1 / 2}
$$

oder, mit $n_{\mathrm{e}}=Z n_{\mathrm{i}}, \quad \tau_{\mathrm{i}}=\left(m_{\mathrm{i}} / m_{\mathrm{e}}\right)^{1 / 2} \tau_{\mathrm{e}} / \sqrt{2} Z^{2}$,

$$
a=P^{\prime \prime 1 / 2}\left(1+\omega_{\mathrm{e}}^{2} \tau_{\mathrm{e}}^{2}\right)^{1 / 2} Z^{-3 / 2}\left(\frac{2}{9} \frac{m_{\mathrm{i}}}{m_{\mathrm{e}}}\right)^{1 / 4} r_{\mathrm{ge}},
$$

wo $r_{\text {ge }}$ der Gyroradius der Elektronen ist. Daraus ergeben sich die Grenzfälle

$$
\begin{aligned}
a & =Z^{-3 / 2}\left(\frac{2}{9} \frac{m_{\mathrm{i}}}{m_{\mathrm{e}}}\right)^{1 / 4} r_{\mathrm{ge}} \quad \text { für } \quad \omega_{\mathrm{e}}^{2} \tau_{\mathrm{e}}^{2} \ll 1, \\
a & =Z^{-3 / 2}\left(\frac{2}{9} \frac{m_{\mathrm{i}}}{m_{\mathrm{e}}}\right)^{1 / 4} \lambda_{\mathrm{e}} \quad \text { für } \quad \omega_{\mathrm{e}}^{2} \tau_{\mathrm{e}}^{2} \gg 1, \quad \text { aber } \quad \omega_{\mathrm{i}}{ }^{2} \tau_{\mathrm{i}}{ }^{2} \ll 1, \\
a & =Z^{1 / 2}\left(\frac{3}{20}\right)^{1 / 2}\left(\frac{2}{9} \frac{m_{\mathrm{i}}}{m_{\mathrm{e}}}\right)^{1 / 4} r_{\mathrm{gi}} \quad \text { für } \quad \omega_{\mathrm{i}}{ }^{2} \tau_{\mathrm{i}}{ }^{2} \gg 1 .
\end{aligned}
$$

Dabei sind $\lambda_{\mathrm{e}}$ die freie Weglänge der Elektronen und $r_{\mathrm{gi}}$ der Gyroradius der Ionen. Abb. 3 zeigt als Beispiel den Verlauf von $a$ als Funktion von $B$ für ein Wasserstoffplasma mit einer Elektronendichte von $n_{\mathrm{e}}=3 \cdot 10^{15} \mathrm{~cm}^{-3}$ und der Temperatur $T=$ $10^{5} \mathrm{~K}$, also für Plasmaparameter, wie sie für eine Bogenentladung in einem Magnetfeld typisch sind. Der Verlauf der Asymptoten hängt nach den Gln.

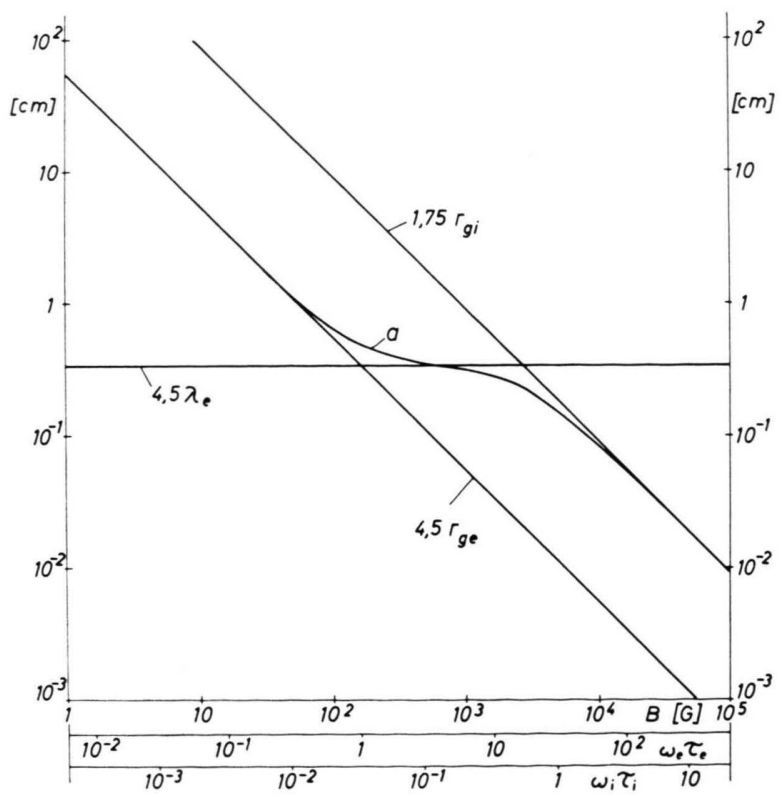

Abb. 3. Die für ein reibend rotierendes Plasma charakteristische Länge $a$ als Funktion von $B$ für ein Wasserstoffplasma mit der Elektronendichte $n_{\mathrm{e}}=3 \cdot 10^{15} \mathrm{~cm}^{-3}$ und der Temperatur $T=10^{5}{ }^{\circ} \mathrm{K}$. Die drei Geraden 4,5 $r_{\text {ge }}, 4,5 \lambda_{\mathrm{e}}$ und $1,75 r_{\mathrm{gi}}$ entsprechen den durch die Gln. $(9 \mathrm{a}),(9 \mathrm{~b})$ und $(9 \mathrm{c})$ gegebenen Grenzfällen. (9a) und (9c) nur von $B$ und $T$ ab: $r_{\mathrm{ge}}, r_{\mathrm{gi}} \sim T^{1 / 2} / B$. Vergrößerung der Dichte verschiebt nur den Bereich, in dem der Übergang zwischen beiden Asymptoten erfolgt, zu höheren Werten von $B$. Die Lage dieses Übergangs ist nach Abb. 3 durch die Gerade $(9 \mathrm{~b})$ gegeben.

\section{Lösungen der Plasmagleichungen}

Es wird jetzt das System (5), (8) der Differentialgleichungen für $V(r, z)$ und $v_{\varphi}(r, z)$ gelöst. Dazu werden die dimensionslosen Koordinaten

$$
\varrho=r / a, \quad \zeta=z / z_{0}
$$

und die dimensionslosen Größen $\hat{V}$ und $\hat{w}$ durch

$$
V=V_{0} \hat{V}, \quad v_{\varphi}=\left(V_{0} / a B\right) \hat{w}
$$

eingeführt. Über die Konstante $V_{0}$ wird später verfügt. Einsetzen in die Gln. (5) und (8) liefert

$$
\begin{array}{r}
\frac{1}{a^{2}}\left[\frac{\partial^{2} \hat{V}}{\partial \varrho^{2}}+\frac{1}{\varrho} \frac{\partial \hat{V}}{\partial \varrho}\right]+\frac{\sigma}{\sigma^{\prime}} \frac{1}{z_{0}^{2}} \frac{\partial^{2} \hat{V}}{\partial \zeta^{2}}=\frac{1}{a^{2}}\left[\frac{\partial \hat{w}}{\partial \varrho}+\frac{\hat{w}}{\varrho}\right], \\
\frac{1}{a^{2}}\left[\frac{\partial^{2} \hat{w}}{\partial \varrho^{2}}+\frac{1}{\varrho} \frac{\partial \hat{w}}{\partial \varrho}-\frac{\widehat{w}}{\varrho^{2}}\right] \\
+\frac{\mu^{\prime}}{\mu^{\prime \prime}} \frac{1}{z_{0}^{2}} \frac{\partial^{2} \hat{w}}{\partial \zeta^{2}}-\frac{\widehat{w}}{a^{2}}=-\frac{1}{a^{2}} \frac{\partial \hat{V}}{\partial \varrho},
\end{array}
$$

oder, mit

$$
\begin{gathered}
\beta^{2}=\frac{\sigma^{\prime}}{\sigma}\left(\frac{z_{0}}{a}\right)^{2}, \\
\gamma^{2}=\frac{\mu^{\prime \prime}}{\mu^{\prime}}\left(\frac{z_{0}}{a}\right)^{2}
\end{gathered}
$$


die Gln.

$$
\begin{gathered}
\frac{\partial^{2} \hat{V}}{\partial \varrho^{2}}+\frac{1}{\varrho} \frac{\partial \hat{V}}{\partial \varrho}+\frac{1}{\beta^{2}} \frac{\partial^{2} \hat{V}}{\partial \zeta^{2}}=\frac{\partial \hat{w}}{\partial \varrho}+\frac{\hat{w}}{\varrho}, \\
\frac{\partial^{2} \widehat{w}}{\partial \zeta^{2}}+\frac{1}{\varrho} \frac{\partial \hat{w}}{\partial \varrho}-\frac{\widehat{w}}{\varrho^{2}}+\frac{1}{\gamma^{2}} \frac{\partial^{2} \widehat{w}}{\partial \zeta^{2}}-\hat{w}=-\frac{\partial \hat{V}}{\partial \varrho} .
\end{gathered}
$$

Der Vergleich dieser beiden GIn. mit der Besselschen Dgl.

$$
\frac{\partial^{2} J_{n}}{\partial \varrho^{2}}+\frac{1}{\varrho} \frac{\partial J_{n}}{\partial \varrho}+\left(k^{2}-\frac{n^{2}}{\varrho^{2}}\right) J_{n}=0 .
$$

legt den Ansatz

$$
\begin{aligned}
& \hat{V}(\varrho, \zeta)=J_{0}(k \varrho) f(\zeta), \\
& \hat{w}(\varrho, \zeta)=J_{1}(k \varrho) g(\zeta)
\end{aligned}
$$

nahe. Dabei sind $J_{0}(k \varrho)$ und $J_{1}(k \varrho)$ die Bessel'schen Funktionen nullter und erster Ordnung. Setzt man diesen Ansatz in die Gln. (12), (13) ein, so erhält man unter Benutzung von

$$
\frac{\partial J_{0}}{\partial(k \varrho)}=-J_{1}, \frac{\partial J_{1}}{\partial(k \varrho)}+\frac{J_{1}}{k \varrho}=J_{0}
$$

und nach Substitution der Bessel'schen Gl. die Gln.

$$
\begin{gathered}
-k^{2} f J_{0}+\frac{1}{\beta^{2}} J_{0} \frac{\partial^{2} f}{\partial \zeta^{2}}=k J_{0} g, \\
-\left(1+k^{2}\right) g J_{1}+\frac{1}{\gamma^{2}} J_{1} \frac{\partial^{2} g}{\partial \zeta^{2}}=k J_{1} f .
\end{gathered}
$$

Der Ansatz separiert also das Problem. Aus den mit Hilfe dieses Ansatzes gewonnenen Partikulärlösungen lassen sich allgemeine Lösungen als Integraldarstellungen nach Bessel-Funktionen aufbauen. Es ist jetzt noch das System

$$
\begin{gathered}
f^{\prime \prime}-k^{2} \beta^{2} f=k \beta^{2} g, \\
g^{\prime \prime}-\left(1+k^{2}\right) \gamma^{2} g=k \gamma^{2} f
\end{gathered}
$$

zu lösen. Dabei bedeuten die Striche Ableitungen nach $\zeta$. Dieses System läßt sich durch Umwandlung in zwei Dgln. vierter Ordnung entkoppeln. Diese lauten:

$$
\begin{aligned}
& f^{\prime \prime \prime \prime}-\left[\gamma^{2}+k^{2}\left(\beta^{2}+\gamma^{2}\right)\right] f^{\prime \prime}+\beta^{2} \gamma^{2} k^{4} f=0, \\
& g^{\prime \prime \prime \prime}-\left[\gamma^{2}+k^{2}\left(\beta^{2}+\gamma^{2}\right)\right] g^{\prime \prime}+\beta^{2} \gamma^{2} k^{4} g=0 .
\end{aligned}
$$

$f$ und $g$ gehorchen also derselben Dgl. vierter Ordnung. Setzt man zur Abkürzung

$$
\begin{gathered}
\gamma^{2}+k^{2}\left(\beta^{2}+\gamma^{2}\right)=2 p, \\
\beta^{2} \gamma^{2} k^{4}=q
\end{gathered}
$$

so führt der Ansatz $f, g=\exp (\lambda \zeta)$ auf

$$
\lambda^{4}-2 p \lambda^{2}+q=0
$$

bzw.

Nun ist

$$
\lambda^{2}=p\left[1 \pm \sqrt{1-\frac{q}{p^{2}}}\right] .
$$

$$
\frac{q}{p^{2}}=\frac{1}{4} \frac{\beta^{2} \gamma^{2} k^{4}}{\left[\gamma^{2}+\left(\beta^{2}+\gamma^{2}\right) k^{2}\right]^{2}}<1 .
$$

Somit hat Gl. (16) die vier reellen Lösungen

$$
\begin{aligned}
& \lambda_{1}=\sqrt{p\left[1+\sqrt{1-q / p^{2}}\right]}, \\
& \lambda_{2}=\sqrt{p\left[1-\sqrt{1-q / p^{2}}\right]}, \\
& \lambda_{3}=-\lambda_{1}, \quad \lambda_{4}=-\lambda_{2} .
\end{aligned}
$$

Wie sich zeigen wird, ist es zweckmäßig, die Exponentialfunktionen $\exp \left(\lambda_{1} \zeta\right), \exp \left(-\lambda_{1} \zeta\right)$ etc. durch die Hyperbelfunktionen zu ersetzen und diese durch ihre Werte bei $\zeta=1 \mathrm{zu}$ dividieren. Damit erhält man

$$
\begin{aligned}
f(\zeta)= & A_{1} \frac{\sinh \lambda_{1} \zeta}{\sinh \lambda_{1}}+B_{1} \frac{\cosh \lambda_{1} \zeta}{\cosh \lambda_{1}} \\
& +A_{2} \frac{\sinh \lambda_{2} \zeta}{\sinh \lambda_{2}}+B_{2} \frac{\cosh \lambda_{2} \zeta}{\cosh \lambda_{2}} \\
g(\zeta)= & C_{1} \frac{\sinh \lambda_{1} \zeta}{\sinh \lambda_{1}}+D_{1} \frac{\cosh \lambda_{1} \zeta}{\cosh \lambda_{1}} \\
& +C_{2} \frac{\sinh \lambda_{2} \zeta}{\sinh \lambda_{2}}+D_{2} \frac{\cosh \lambda_{2} \zeta}{\cosh \lambda_{2}}
\end{aligned}
$$

Die acht Koeffizienten $A_{1}, \ldots, D_{2}$ unterliegen zunächst einmal der Bedingung, daß $f(\zeta)$ und $g(\zeta)$ auch Lösungen des gekoppelten Systems (14), (15) sein müssen. Setzt man $f$ und $g$ in diese Gln. ein, so findet man wegen der linearen Unabhängigkeit der Summanden in $f$ und $g$ die Beziehungen

$$
\begin{gathered}
\left(\lambda_{1}^{2}-k^{2} \beta^{2}\right) A_{1}=k \beta^{2} C_{1}, \\
{\left[\lambda_{1}^{2}-\left(1+k^{2}\right) \gamma^{2}\right] C_{1}=k \gamma^{2} A_{1}}
\end{gathered}
$$

und analoge Beziehungen für die drei anderen Koeffizientenpaare $\left(A_{2}, C_{2}\right),\left(B_{1}, D_{1}\right)$ und $\left(B_{2}, D_{2}\right)$. Bildet man die Determinanten dieser Gleichungssysteme, so erhält man für diese $\lambda_{1,2}^{4}-2 p \lambda_{1,2}^{2}+q$. Nach Gl. (16) verschwinden die Determinanten. Die Gleichungssysteme für die Koeffizientenpaare haben also eine einparametrige Schar von Lösungen, d.h. durch die Berücksichtigung der Kopplung werden vier der acht Koeffizienten eliminiert. Die weiteren Bestimmungsgleichungen werden durch die Randbedingungen geliefert.

Die Rotationsgeschwindigkeit soll für $z= \pm z_{0}$, bzw. für $\zeta= \pm 1$ verschwinden, und zwar unabhängig von der speziellen Form der Elektroden. Das ist nur dann gewährleistet, wenn alle Partikulärlösungen $J_{1}(k \varrho) g(k, \zeta)$ für $\zeta= \pm 1$ verschwin- 
den. Somit ist $g(k, 1)=0, g(k,-1)=0 \mathrm{zu}$ fordern, woraus

$$
g(1)=C_{1}+C_{2}+D_{1}+D_{2}=0, \quad g(-1)=-C_{1}-C_{2}+D_{1}+D_{2}=0,
$$

oder $C_{2}=-C_{1}=-C, D_{2}=-D_{1}=-D$ folgt. Somit ist

$$
g(\zeta)=C\left(\frac{\sinh \lambda_{1} \zeta}{\sinh \lambda_{1}}-\frac{\sinh \lambda_{2} \zeta}{\sinh \lambda_{2}}\right)+D\left(\frac{\cosh \lambda_{1} \zeta}{\cosh \lambda_{1}}-\frac{\cosh \lambda_{2} \zeta}{\cosh \lambda_{2}}\right)
$$

Da die Verhältnisse $A_{1} / C_{1}, A_{2} / C_{2}$ etc. durch die Kopplungsbedingungen gegeben sind, ergibt sich daraus weiter

$$
\begin{aligned}
f(\zeta)= & k \beta^{2} C\left(\frac{1}{\lambda_{1}{ }^{2}-k^{2}} \frac{\sinh \lambda_{1} \lambda^{2}}{\sinh \lambda_{1}}-\frac{1}{\lambda_{2}{ }^{2}-k^{2} \beta^{2}} \frac{\sinh \lambda_{2} \zeta}{\sinh \lambda_{2}}\right) \\
& +k \beta^{2} D\left(\frac{1}{\lambda_{1}{ }^{2}-k^{2} \beta^{2}} \frac{\cosh \lambda_{1} \zeta}{\cosh \lambda_{1}}-\frac{1}{\lambda_{2}{ }^{2}-k^{2} \beta^{2}} \frac{\cosh \lambda_{2} \zeta}{\cosh \lambda_{2}}\right)
\end{aligned}
$$

Die Funktionen $f(k, \zeta) J_{0}(k \varrho)$ und $g(k, \zeta) J_{1}(k \varrho)$ sind Partikulärlösungen des Systems (12), (13), wobei $g(k, \zeta)$ bereits den Randbedingungen $\hat{w}(\zeta= \pm 1)=0$ genügt. Da $J_{0}(k \varrho)$ und $J_{1}(k \varrho)$ für $\varrho \rightarrow \infty$ verschwinden und außerdem $J_{1}(0)=0$ gilt, erfüllen die Partikulärlösungen auch schon die Randbedingungen bezüglich $\varrho$. Es gibt jedoch offensichtlich keine Partikulärlösung derart, daß $\hat{V}$ und $\partial \hat{V} / \partial \zeta \sim j_{z}$ das für $\zeta= \pm 1$ vorgeschriebene Verhalten haben. Das kann nur von der allgemeinen Lösung geleistet werden, die als Integraldarstellung ${ }^{5}$

$$
\begin{aligned}
& \hat{V}(\varrho, \zeta)=\int_{0}^{\infty} f(k, \zeta) J_{0}(k \varrho) k \mathrm{~d} k, \\
& \hat{w}(\varrho, \zeta)=\int_{0}^{\infty} g(k, \zeta) J_{1}(k \varrho) k \mathrm{~d} k
\end{aligned}
$$

angegeben werden kann. Dabei sind die in den Funktionen $f$ und $g$ auftretenden Koeffizienten $C$ und $D$ ebenfalls als Funktionen von $k$ aufzufassen. Diese Funktionen $C(k)$ und $D(k)$ sind jetzt so zu bestimmen, daß $\hat{V}$ auf den Elektroden konstant ist, und daß $\partial \hat{V} / \partial \zeta$ in den Ebenen $\zeta= \pm 1$ außerhalb der Elektroden verschwindet. Dieses gemischte Randwertproblem führt im allgemeinen zu Integralgleichungen für $C(k)$ und $D(k)$; es vereinfacht sich jedoch, wenn man Elektroden ohne radiale Ausdehnung, d.h. Punkte oder Kreislinien betrachtet.
In diesem Fall ist die Bedingung, daß $\hat{V}$ auf den Elektroden konstant sein muß, wegen der Rotationssymmetrie trivialerweise erfüllt. Dann läßt sich das Problem geschlossen lösen. Dieser ganz erheblichen mathematischen Vereinfachung wegen werden die folgenden Untersuchungen zunächst auf diesen Fall beschränkt. Aus der Diskussion der Resultate lassen sich aber schon Rückschlüsse auf das Verhalten von Lichtbögen mit Elektroden endlicher radialer Ausdehnung ziehen.

Für radial nicht ausgedehnte Elektroden $\left(r_{k}=\right.$ $\left.R_{\mathrm{K}}, r_{\mathrm{A}}=R_{\mathrm{A}}\right) \mathrm{mu}$ die Stromdichte und damit auch $\partial \hat{V} / \partial \zeta$ auf den Elektroden den Charakter einer $\delta$-Funktion haben. Sei $\varrho_{\mathrm{A}}=R_{\mathrm{A}} / a$ der reduzierte Radius der Anode, $\varrho_{\mathbf{K}}$ der reduzierte Radius der Kathode, so lauten die Randbedingungen für punkt- oder kreislinienförmige Elektroden

$$
\begin{aligned}
& -\left.\frac{\partial \hat{V}}{\partial \zeta}\right|_{\zeta=-1}=\delta\left(\varrho-\varrho_{\mathrm{A}}\right), \\
& -\left.\frac{\partial \hat{V}}{\partial \zeta}\right|_{\zeta=1}=\delta\left(\varrho-\varrho_{\mathrm{K}}\right) .
\end{aligned}
$$

Wählt man nun für die $\delta$-Funktion die Darstellung 5

$$
\delta\left(\varrho-\varrho_{0}\right)=\int_{0}^{\infty} J_{0}(k \varrho) J_{0}\left(k \varrho_{0}\right) k \partial k,
$$

so erhält man

$$
\begin{aligned}
& -\left.\frac{\partial \hat{V}}{\partial \zeta}\right|_{\zeta=-1}=-\left.\int_{0}^{\infty} \frac{\partial f}{\partial \zeta}\right|_{\zeta=1} J_{0}(k \varrho) k \mathrm{~d} k=\int_{0}^{\infty} J_{0}\left(k \varrho_{\mathrm{A}}\right) J_{0}(k \varrho) k \mathrm{~d} k, \\
& -\left.\frac{\partial \hat{V}}{\partial \zeta}\right|_{\zeta=-1}=-\left.\int_{0}^{\infty} \frac{\partial f}{\partial \zeta}\right|_{\zeta=1} J_{0}(k \varrho) k \mathrm{~d} k=\int_{0}^{\infty} J_{0}\left(k \varrho_{\mathrm{K}}\right) J_{0}(k \varrho) k \mathrm{~d} k .
\end{aligned}
$$

5 Vgl. z. B. A. Sommerfeld : Vorlesungen über theoretische Physik, Bd. VI, Springer-Verlag, Berlin 1947. 
Da diese Beziehungen für alle $\varrho$ gelten, müssen in beiden Gln. die Integranden auf beiden Seiten gleich sein. Diese beiden Gln. sind somit Bestimmungsgleichungen für $C(k)$ und $D(k)$. Durch Einsetzen von $\partial f / \partial \zeta$ an den Stellen $\zeta=-1$ und $\zeta=1$ erhält man nach einigen Umformungen die Lösung

$$
\begin{aligned}
\hat{V}(\varrho, \zeta)=- & \frac{1}{2} \int_{0}^{\infty} J_{0}(k \varrho)\left[\left(J_{0}\left(k \varrho_{\mathrm{K}}\right)+J_{0}\left(k \varrho_{\mathrm{A}}\right)\right) \frac{\left(\lambda_{2}{ }^{2}-k^{2} \beta^{2}\right) \frac{\sinh \lambda_{1} \zeta}{\sinh \lambda_{1}}-\left(\lambda_{1}{ }^{2}-k^{2} \beta^{2}\right) \frac{\sinh \lambda_{2} \zeta}{\sinh \lambda_{2}}}{\left(\lambda_{2}{ }^{2}-k^{2} \beta^{2}\right) \lambda_{1} \operatorname{ctgh} \lambda_{1}-\left(\lambda_{1}^{2}-k^{2} \beta^{2}\right) \lambda_{2} \operatorname{ctgh} \lambda_{2}}\right. \\
& +\left(\left(J_{0}\left(k \varrho_{\mathrm{K}}\right)-J_{0}\left(k \varrho_{\mathrm{A}}\right)\right) \frac{\left(\lambda_{2}{ }^{2}-k^{2} \beta^{2}\right) \frac{\cosh \lambda_{1} \zeta}{\cosh \lambda_{1}}-\left(\lambda_{1}{ }^{2}-k^{2} \beta^{2}\right) \frac{\cosh \lambda_{2} \zeta}{\cosh \lambda_{2}}}{\left(\lambda_{2}{ }^{2}-k^{2} \beta^{2}\right) \lambda_{1} \operatorname{tgh} \lambda_{1}-\left(\lambda_{1}{ }^{2}-k^{2} \beta^{2}\right) \lambda_{2} \operatorname{tgh} \lambda_{2}}\right] k \mathrm{~d} k
\end{aligned}
$$

und daraus weiter

$$
\begin{aligned}
w(\varrho, \zeta)= & -\frac{1}{2} \int_{0}^{\infty} \frac{1}{k \beta^{2}} J_{1}(k \varrho)\left[\frac{\left(\lambda_{1}^{2}-k^{2} \beta^{2}\right)\left(\lambda_{2}^{2}-k^{2} \beta^{2}\right)\left(\frac{\sinh \lambda_{1} \zeta}{\sinh \lambda_{1}}-\frac{\sinh \lambda_{2} \zeta}{\sinh \lambda_{2}}\right)}{\left(\lambda_{2}^{2}-k^{2} \beta^{2}\right) \lambda_{1} \operatorname{ctgh} \lambda_{1}-\left(\lambda_{1}^{2}-k^{2} \beta^{2}\right) \lambda_{2} \operatorname{ctgh} \lambda_{2}}\left(J_{0}\left(k \varrho_{\mathrm{K}}\right)+J_{0}\left(k \varrho_{\mathrm{A}}\right)\right)\right. \\
& \left.+\frac{\left(\lambda_{1}^{2}-k^{2} \beta^{2}\right)\left(\lambda_{2}{ }^{2}-k^{2} \beta^{2}\right)\left(\frac{\cosh \lambda_{1} \zeta}{\cosh \lambda_{1}}-\frac{\cosh \lambda_{2} \zeta}{\cosh \lambda_{2}}\right)}{\left(\lambda_{2}{ }^{2}-k^{2} \beta^{2}\right) \lambda_{1} \operatorname{tgh} \lambda_{1}-\left(\lambda_{1}^{2}-k^{2} \beta^{2}\right) \lambda_{2} \operatorname{tgh} \lambda_{2}}\left(J_{0}\left(k \varrho_{\mathrm{K}}\right)-J_{0}\left(k \varrho_{\mathrm{A}}\right)\right)\right] k \mathrm{~d} k
\end{aligned}
$$

Aus Gl. (18) lassen sich durch Differentiation die Größen $-\partial \hat{V} \mid \partial \varrho \sim E_{\mathrm{r}}$ und $-\partial \hat{V} \mid \partial \zeta \sim E_{z}=j_{z} / \sigma$ bestimmen. Infolge der Annahme $\sigma=$ const ist $-\partial \hat{V} \mid \partial \zeta$ auch zu $j_{z}$ proportional.

Die Gln. (18) und (19) sind die exakten Lösungen des Systems (12), (13) für den Fall radial nicht ausgedehnter Elektroden. Wegen der Kompliziertheit der Integrale wurde die Auswertung der Gln. (18) und (19) maschinell durchgeführt. Bevor einige typische Resultate präsentiert werden, soll auf einige allgemeine Eigenschaften der Lösungen hingewiesen werden.

\section{(1) Normierung}

Die gewählte Darstellung der $\delta$-Funktion ist so normiert, daß

$$
\int_{0}^{\infty} \delta\left(\varrho-\varrho_{\mathrm{K}}\right) \varrho \mathrm{d} \varrho=1, \quad \int_{0}^{\infty} \delta\left(\varrho-\varrho_{\mathrm{A}}\right) \varrho \mathrm{d} \varrho=1
$$

gilt. Da $-\partial \hat{V} \mid \partial \zeta$ an den Elektroden in diese $\delta$ Funktionen übergeht und da der Gesamtstrom durch jede Ebene der gleiche ist, gilt für alle $\zeta$

$$
-\int_{0}^{\infty} \frac{\partial \hat{V}}{\partial \zeta} \varrho \mathrm{d} \varrho=1
$$

In den ursprünglichen Koordinaten gilt entsprechend

$$
2 \pi \int_{0}^{\infty} j_{z} r \mathrm{~d} r=-2 \pi \sigma \int_{0}^{\infty} \frac{\partial V}{\partial z} r \mathrm{~d} r=I,
$$

wo $I$ der Bogenstrom ist. Vergleich dieser beiden Gln. liefert für die noch nicht bestimmte Konstante $V_{0}$ den Wert

$$
V_{0}=\frac{z_{0} I}{2 \pi a^{2} \sigma} .
$$

\section{(2) Konvergenzverhalten}

Gemäß den Randbedingungen divergiert $\partial \hat{V} \mid \partial \zeta$ in den Ebenen $\zeta=1$ und $\zeta=-1$ bei den Radien $\varrho_{\mathrm{K}}$ und $\varrho_{\mathrm{A}}$. Durch Entwicklung der Integranden in (18) und (19) nach großen $k$ läßt sich zeigen, daß an diesen Stellen auch $\hat{V}$ und $\partial \hat{V} \mid \partial \varrho$ divergieren, wie es auch physikalisch sinnvoll ist. Auch $\hat{w}$ ist in den Ebenen $\zeta= \pm 1$ für $\varrho=\varrho_{\mathrm{K}}$ und $\varrho=\varrho_{\mathrm{A}}$ singulär. Das folgt wegen der Singularität von $\partial \hat{V} \mid \partial \varrho$ an diesen Stellen aus Gl. (13). Aus der Entwicklung der Integranden von $\hat{V}, \partial \hat{V}|\partial \varrho, \partial \hat{V}| \partial \zeta$ und $\hat{w}$ nach großen $k$ läßt sich ebenso zeigen, daß diese Funktionen überall mit Ausnahme der Elektroden regulär sind, sowie, daß auch $\hat{V}$ und $\hat{u}$ ' ebenso wie die Partikulärlösungen für $\varrho \rightarrow \infty$ verschwinden. Die Entwicklung der Integranden in (18) und (19) nach großen $k$ liefert ferner Abschätzungen dafür, bei welchem Wert $k_{\max }$ die Integration abgebrochen werden darf. Da $\hat{V}$ und die Ableitungen von $\hat{V}$ für $\varrho=\varrho_{\mathrm{A}}, \zeta=-1$ und $\varrho=\varrho_{\mathrm{K}}, \zeta=1$ divergieren, ist $k_{\max }$ bei festem $\varrho$ um so größer, je größer $|\zeta|$ ist. Weiter folgt aus den Eigenschaften der BesselFunktionen, daß $k_{\max }$ bei festem $\zeta$ um so größer ist, je größer $\varrho$ ist. Einen einfachen Test dafür, ob $k_{\max }$ hinreichend groß gewählt wurde, liefert die Normierungsbedingung (20). Danach muß für hinreichend große $\varrho^{*}$

$$
1-\int_{0}^{\varrho^{*}}\left(-\begin{array}{c}
\partial \hat{V} \\
\partial \zeta
\end{array}\right) \varrho d \varrho \equiv 1-F\left(\varrho^{*}\right) \ll 1
$$

gelten. Dieser Test wurde immer durchgeführt. Eine Verletzung dieser Beziehung konnte stets durch 
Vergrößerung des Wertes $k_{\max }$, bei dem die Integration über $k$ abgebrochen wird, behoben werden.

\section{(3) Symmetrieeigenschaften}

Ein symmetrischer Lichtbogen liegt vor, wenn die zweiten Terme der Gln. (18) und (19). Aus den Symmetrieeigenschaften der Funktionen $\sinh x$ und $\cosh x$ folgen dann die Symmetrieeigenschaften

$$
\begin{gathered}
\hat{V}(\varrho,-\zeta)=-\hat{V}(\varrho, \zeta), \\
\hat{w}(\varrho,-\zeta)=-\hat{w}(\varrho, \zeta), \\
\frac{\partial \hat{V}}{\partial \varrho}(\varrho,-\zeta)=-\frac{\partial \hat{V}}{\partial \varrho}(\varrho, \zeta), \\
\frac{\partial \hat{V}}{\partial \zeta}(\varrho,-\zeta)=+\frac{\partial \hat{V}}{\partial \zeta}(\varrho, \zeta) .
\end{gathered}
$$

$\hat{V}, \partial \hat{V} / \partial \varrho$ und $\hat{w}$ verschwinden somit in der Ebene $\zeta=0$. Ebenso verschwindet dort $\partial^{2} \hat{V} / \partial \zeta^{2}$, d.h. in der Mittelebene der Anordnung ist die zu $\partial \hat{V} / \partial \zeta$ proportionale Stromdichteverteilung am breitesten. Alle diese Eigenschaften sind auch von der Anschauung her zu erwarten. $\varrho_{\mathrm{K}}=\varrho_{\mathrm{A}}$ ist. In diesem Fall verschwinden jeweils

\section{(4) Parameter der Lösungen}

Die Lösungen (18) und (19) hängen von den vier Parametern $\varrho_{\mathrm{A}}, \varrho_{\mathrm{K}}, \beta$ und $\gamma$ ab. Die Abhängigkeit dieser Parameter vom Magnetfeld und den Plasmaparametern soll jetzt diskutiert werden, um Anhaltspunkte für die Auswahl charakteristischer Werte dieser Parameter zu gewinnen.

$\varrho_{\mathrm{A}}$ und $\varrho_{\mathrm{K}}$ sind die durch $a$ dividierten Elektrodenradien. Da $a$ ein Maß für die Verbreiterung der Stromdichteverteilung ist, ist $\mathrm{zu}$ erwarten, daß wesentliche Unterschiede zwischen punkt- und kreislinienförmigen Elektroden nur für $\varrho_{\mathrm{A}}, \varrho_{\mathrm{K}} \gg 1$ auftreten. Da $a$ monoton mit $B$ abnimmt (vgl. dazu Abb. 3), nehmen $\varrho_{\mathrm{A}}$ und $\varrho_{\mathrm{K}}$ monoton mit $B$ zu, d.h. der Einfluß der Elektrodengeometrie ist in den elektrodenfernen Gebieten um so größer, je größer das Magnetfeld ist. Das entspricht dem, was man von der Anschauung her erwartet.

In die Parameter $\beta$ und $\gamma$ geht der halbe Elektrodenabstand $z_{0}$ ein:

$$
\begin{aligned}
\beta^{2}=\frac{\sigma^{\prime}}{\sigma}\left(\frac{z_{0}}{a}\right)^{2} & =\frac{1}{1+\omega_{\mathrm{e}}^{2}} \overline{\tau_{\mathrm{e}}^{2}}\left(\frac{z_{0}}{a}\right)^{2}, \\
\gamma^{2} & =\frac{\mu^{\prime \prime}}{\mu^{\prime}}\left(\frac{z_{0}}{a}\right)^{2} .
\end{aligned}
$$

Für $\beta$ erhält man unter Zuhilfenahme der Gl. (9a) bis (9c) die asymptotischen Ausdrücke

$$
\begin{aligned}
& \beta=Z^{3 / 2}\left(\frac{9}{2} \frac{m_{\mathrm{e}}}{m_{\mathrm{i}}}\right)^{1 / 4} \frac{z_{0}}{r_{\mathrm{ge}}} \text { für } \omega_{\mathrm{e}}^{2} \tau_{\mathrm{e}}^{2} \ll 1, \\
& \beta=Z^{3 / 2}\left(\frac{9}{2} \frac{m_{\mathrm{i}}}{m_{\mathrm{e}}}\right)^{1 / 4} \frac{z_{0}}{\lambda_{\mathrm{e}}} \frac{1}{\omega_{\mathrm{e}} \tau_{\mathrm{e}}} \text { für } \omega_{\mathrm{e}}^{2} \tau_{\mathrm{e}}^{2} \gg 1, \text { aber } \omega_{\mathrm{i}}{ }^{2} \tau_{\mathrm{i}}{ }^{2} \ll 1, \\
& \beta=Z^{3 / 2}\left(\frac{10}{3}\right)^{1 / 2}\left(\frac{9}{2}\right)^{1 / 4}\left(\frac{m_{\mathrm{e}}}{m_{\mathrm{i}}}\right)^{3 / 4} \frac{z_{0}}{\lambda_{\mathrm{e}}} \text { für } \omega_{\mathrm{i}}^{2} \tau_{\mathrm{i}}{ }^{2} \gg 1 .
\end{aligned}
$$

$\beta$ wächst demnach für $\omega_{\mathrm{e}}^{2} \tau_{\mathrm{e}^{2}} \ll 1$ linear mit $B$ an, nimmt für den Fall $(10 \mathrm{~b})$ wie $1 / B$ ab und erreicht für $\omega_{\mathrm{i}}^{2} \tau_{\mathrm{i}}^{2} \gg 1$ einen von $B$ unabhängigen asymptotischen Wert. Somit hat $\beta$ als Funktion von $B$ ein Maximum; aus Gl. (10) findet man, daß dieses Maximum bei $\omega_{\mathrm{e}} \tau_{\mathrm{e}}=1$ liegt. Abb. 4 zeigt $\beta$ als Funktion von $B$ für $n_{\mathrm{e}}=3 \cdot 10^{15}, T=10^{5} \mathrm{~K}$, also für dieselben Plasmaparameter, für die der in Abb. 3 gezeigte Verlauf von $a(B)$ berechnet wurde. Der halbe Elektrodenabstand wurde für Abb. 4 mit $z_{0}=5,55 \mathrm{~cm}$ gerade so gewählt, daß $\beta=1$ für $\omega_{\mathrm{i}}{ }^{2} \tau_{\mathrm{i}}{ }^{2} \gg 1$ wird. Für $B=10 \mathrm{G}$ ist ebenfalls $\beta=1$. Für dieses Magnetfeld ist $\omega_{\mathrm{e}}^{2} \tau_{\mathrm{e}}^{2} \ll 1$. Das Maximum von $\beta$ beträgt 8,15 und liegt bei $B=160 \mathrm{G}$. Das Regime $B>10 \mathrm{G}$ umfaßt also die drei Fälle der Gln. (10a) bis (10c). $\beta$ ändert sich in diesem Bereich um weniger als eine Größenordnung.
Abb. 4. Die Parameter $\beta$ und $\gamma$ als Funktion von $B$ für ein Wasserstoffplasma mit der Elektronendichte $n_{\mathrm{e}}=$ $3 \cdot 10^{15} \mathrm{~cm}^{-3}$ und der Temperatur $T=10^{5}{ }^{\circ} \mathrm{K}$. Der Elektrodenabstand wurde mit $2 z_{0}=11,1 \mathrm{~cm}$ so gewählt, daß $\beta=1$ für $\omega_{1}^{2} \tau_{1}^{2} \gg 1$ gilt.

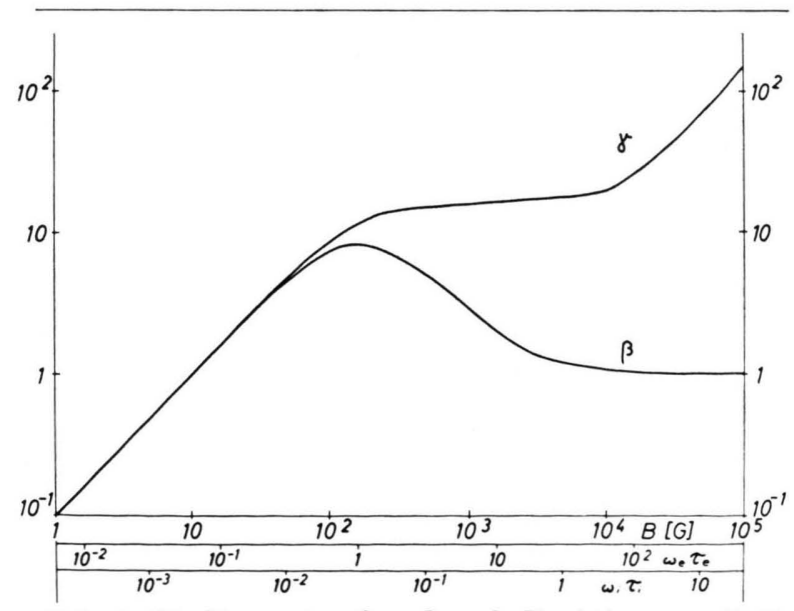


Das Verhalten von $\gamma$ ergibt sich unmittelbar aus Gl. (11). Für $\omega_{\mathrm{i}}{ }^{2} \tau_{\mathrm{i}}{ }^{2} \ll 1$ ist $\mu^{\prime \prime}=\mu^{\prime}$ und somit $\gamma=z_{0} / a$, für $\omega_{\mathrm{i}}{ }^{2} \tau_{\mathrm{i}}{ }^{2} \gg 1$ ist $\mu^{\prime \prime} / \mu^{\prime}=1 / 4$, also $\gamma=z_{0} / 4 a$. $\gamma$ wächst somit monoton mit $B$, und zwar für $\omega_{\mathrm{e}}^{2} \tau_{\mathrm{e}}^{2} \ll 1$ und für $\omega_{\mathrm{i}}{ }^{2} \tau_{\mathrm{i}}{ }^{2} \gg 1$ linear mit $B . \gamma$ ist ebenfalls in Abb. 4 als Funktion von $B$ für die gleichen Plasmaparameter wie bisher aufgetragen.

\section{Typische Resultate für einen zwischen Punktelektroden brennenden Bogen}

Für Punktelektroden hängen die Lösungen (18), (19) nur von den beiden Parametern $\beta$ und $\gamma$ ab. Dieser Fall ist deshalb der übersichtlichste. Er gestattet zudem einen Vergleich mit den Verhältnissen in einem Plasma mit unendlicher Ionenmasse, in dem die Verbreiterung der Stromdichte allein durch den Hall-Effekt bestimmt wird. Die obigen Diskussionen des Verhaltens von $\beta$ und $\gamma$ als Funktionen von $B$ legt nahe, die vier Fälle $\omega_{\mathrm{e}}{ }^{2} \tau_{\mathrm{e}}{ }^{2} \ll 1$; $\omega_{\mathrm{e}}^{2} \tau_{\mathrm{e}}^{2}=1 ; \quad \omega_{\mathrm{e}}^{2} \tau_{\mathrm{e}}^{2} \gg 1, \quad$ aber $\quad \omega_{\mathrm{i}}^{2} \tau_{\mathrm{i}}{ }^{2} \ll 1 ;$ $\omega_{\mathrm{e}}{ }^{2} \tau_{\mathrm{e}}{ }^{2} \gg 1$ und $\omega_{\mathrm{i}}{ }^{2} \tau_{\mathrm{i}}{ }^{2} \gg 1 \mathrm{zu}$ betrachten. Für die bisher zugrunde gelegten Plasmaparameter $n_{\mathrm{e}}=$ $3 \cdot 10^{15} \mathrm{~cm}^{-3}, T=10^{5} \mathrm{~K}$ werden deshalb die vier in Tab. 1 aufgeführten Werte von $B$ gewählt. Diese Tabelle enthält außerdem $\beta$ und $\gamma$ für $z_{0}=5,55 \mathrm{~cm}$.

Die Abb. 5 a bis 5 d zeigen die Stromdichte $j_{z}$ in der Mittelebene $z=0$ des Bogens, wo die Stromverteilung am stärksten verbreitet ist, für die in Tab. 1 angegebenen Magnetfelder und für den Elektrodenabstand $2 z_{0}=11,1 \mathrm{~cm}$. Der Bogenstrom wurde zu $I=1 \mathrm{kA}$ angenommen. Zum Vergleich wurden in dieser Abb. für denselben Bogenstrom die aus Gl. (7) berechneten Stromdichten $j_{z}^{(0)}(0)$ aufgetragen. Am rechten Rand jeder Abb. ist außerdem eine $E_{z}$-Skala angebracht. Unter der Abszisse sind jeweils drei ausgezeichnete Radien eingetragen: $r=a$, wo $a$ die durch Gl. (9) definierte für ein reibend rotierendes Plasma charakteristische Länge ist, ferner $r=2 z_{0}^{\prime}$, wo $z_{0}^{\prime}=z_{0} / \sqrt{1+\omega_{\mathrm{e}}^{2} \tau_{\mathrm{e}}{ }^{2}}$ ist. Die Stromdichte $j_{z}^{(0)}(0)$ ist bei $r=2 z_{0}^{\prime}$ nach Gl. (6) auf ein Achtel des Achsenwertes abgefallen. Vergleichshalber soll der Radius $b$ durch

$$
j_{z}(b, 0)=\frac{1}{8} j_{z}(0,0)
$$

definiert werden. $r=b$ ist ebenfalls auf der $\mathrm{Ab}$ szisse eingezeichnet. Die Stromdichteverteilungen für den zehnfachen Elektrodenabstand, nämlich für $2 z_{0}=111 \mathrm{~cm}$, sind in den Abb. $6 \mathrm{a}$ bis $6 \mathrm{~d}$ dargestellt.

Es fällt auf, daß $j_{z}$ in einigen Fällen für größere Radien auch negative Werte annimmt; besonders deutlich ist dies auf den Abb. $6 \mathrm{c}$ und $6 \mathrm{~d}$ zu sehen. Es konnte sichergestellt werden, daß derartige Verläufe von $j_{z}$ nicht auf Ungenauigkeiten der Rechnung beruhen. Bezeichnet man mit $r_{1}$ den Radius des Nulldurchgangs von $j_{z}$, so ist

$$
2 \pi \int_{0}^{r_{1}} j_{z} r \mathrm{~d} r>I
$$

jedoch für $r^{*} \gg r_{1}$ wieder

$$
2 \pi \int_{0}^{r *} j_{z} r \mathrm{~d} r<I, \quad I-2 \pi \int_{0}^{r *} j_{z} r \mathrm{~d} r \ll I
$$

in Übereinstimmung mit Gl. (22). Eine Erklärung für das Auftreten negativer $j_{z}$-Werte wird anhand weiterer Resultate gegeben.

Der Vergleich der acht Stromdichteverteilungen $j_{z}$ in den Abb. 5 a bis $6 \mathrm{~d}$ untereinander ergibt, daß ihre Breite $b$ bei festem Elektrodenabstand mit wachsendem Magnetfeld abnimmt, wie auch nicht anders zu erwarten. Der Vergleich mit den entsprechenden Verteilungen $j_{z}^{(0)}$ zeigt, daß der Bogenstrom im rotierenden Plasma immer erheblich, in manchen Fällen ganz wesentlich stärker auf die Achsenumgebung konzentriert ist, als im nicht rotierenden Plasma. Will man die Abweichungen quantitativ erfassen, so bieten sich zwei Größen als Maß an, nämlich das Verhältnis $j_{z}(0) / j_{z}^{(0)}(0)$ der Achsenwerte und das Verhältnis $b / 2 z_{0}$ der Achtelwertsbreiten. Diese Größen sind in den Tab. 2 und 3 aufgeführt.

\begin{tabular}{clcccc}
\hline$B$ & $\begin{array}{c}a \\
{[\mathrm{~cm}]}\end{array}$ & $\omega_{\mathrm{e}}^{2} \tau_{\mathrm{e}}^{2}$ & $\omega_{1}^{2} \tau_{1}^{2}$ & $\begin{array}{c}\beta \\
\left(z_{0}=5,55 \mathrm{~cm}\right)\end{array}$ & $\left(z_{0}=5,55 \mathrm{~cm}\right)$ \\
\hline $10 \mathrm{G}$ & 5,55 & $4 \cdot 10^{-3}$ & $\ll 1$ & 1 & 1 \\
$160 \mathrm{G}$ & 0,475 & 1 & $\ll 1$ & 8,15 & 11,5 \\
$1 \mathrm{kG}$ & 0,317 & 40 & $4,5 \cdot 10^{-2}$ & 2,91 & 17,4 \\
$30 \mathrm{kG}$ & $2,85 \cdot 10^{-2}$ & $3,6 \cdot 10^{4}$ & 40 & 1 & 100 \\
\hline
\end{tabular}

Tab. 1. Auswahl typischer Werte für das Magnetfeld. 

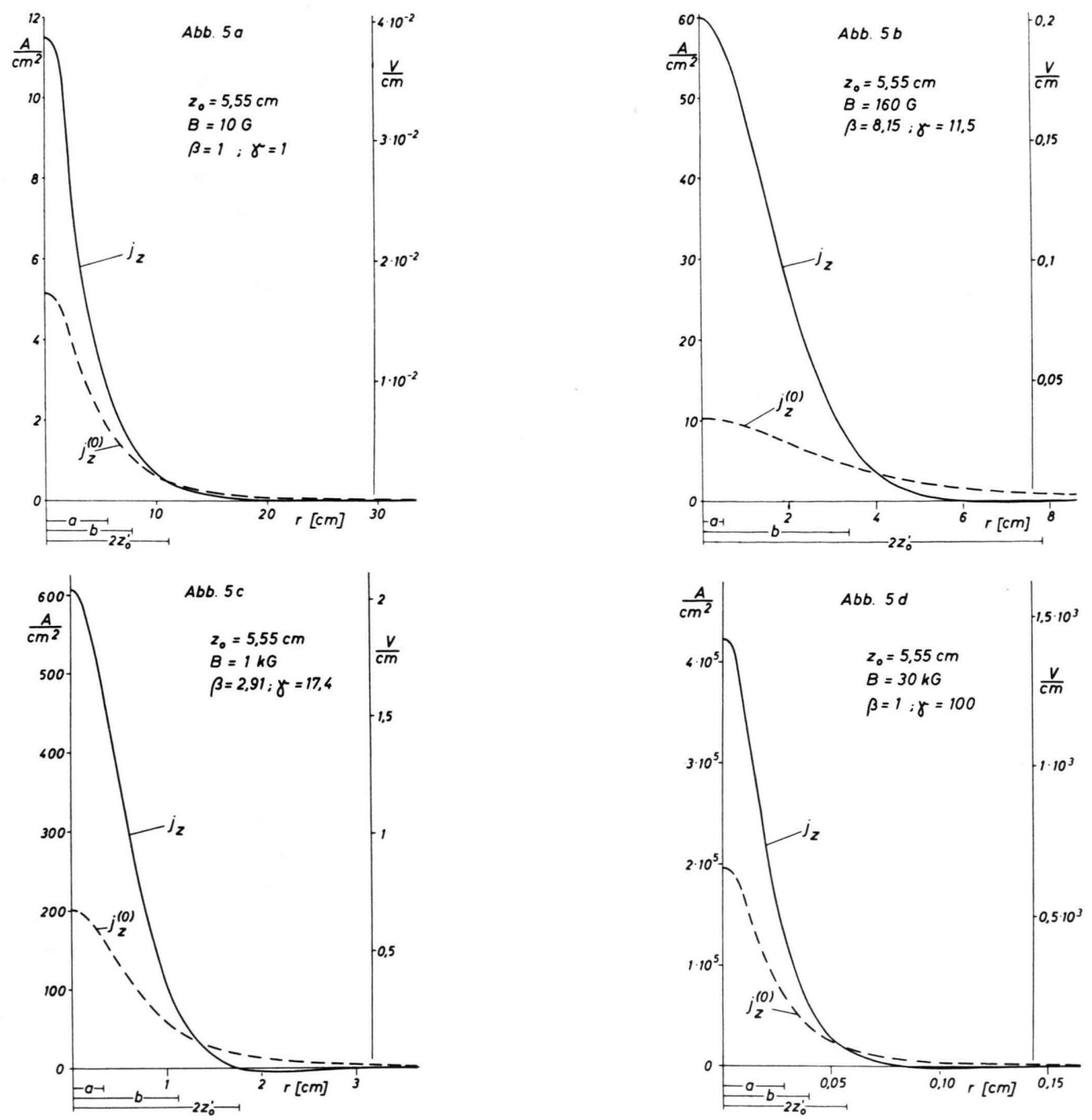

Abb. 5 a bis 5 d. Stromdichten $j_{z}$ im rotierenden Plasma (ausgezogene Kurven) und $j_{z}^{(0)}$ in nicht rotierendem Plasma (gestrichelte Kurven) in der Mittelebene eines zwischen zwei Punktelektroden brennenden Bogens. Wasserstoffplasma, $n_{\mathrm{e}}=3 \cdot 10^{15} \mathrm{~cm}^{-3}, T=10^{15}{ }^{\circ} \mathrm{K}$. Elektrodenabstand $2 z_{0}=11,1 \mathrm{~cm}$. Die Werte für $B$ sind die in Tab. 1 angegebenen. Die Skala am rechten Rand gibt die zugehörigen $E_{z}$-Werte an. Auf der Abszisse sind die charakteristische Länge $a$, die Achtelwertsbreite $2 z_{0}^{\prime}$ für $\mathrm{j}_{z}^{(0)}$ und die Achtelwertsbreite $b$ für $j_{z}$ angegeben. Der Bogenstrom beträgt $1 \mathrm{kA}$.

\begin{tabular}{clclc}
\hline & \multicolumn{2}{c}{$z_{0}=5,55 \mathrm{~cm}$} & \multicolumn{2}{c}{$z_{0}=55,5 \mathrm{~cm}$} \\
$B$ & $\beta$ & $j_{z}(0) / j_{z}^{(0)}(0)$ & \multicolumn{1}{c}{$\beta$} & $j_{z}(0) / j_{z}^{(0)}(0)$ \\
\hline $10 \mathrm{G}$ & 1 & 2,2 & 10 & 6,1 \\
$160 \mathrm{G}$ & 8,15 & 5,8 & 81,5 & 47,7 \\
$1 \mathrm{kG}$ & 2,91 & 3,0 & 29,1 & 20,2 \\
$30 \mathrm{kG}$ & 1 & 2,2 & 10 & 8,8 \\
\hline
\end{tabular}

Tab. 2. Achsenwertverhältnis $j_{z}(0) / j_{z}^{(0)}(0)$ der Stromdichten in rotierenden und nicht rotierenden Plasmen.

Aus den Tab. 2 und 3 geht hervor, daß die Abweichung zwischen rotierendem und nicht rotierendem Plasma umso größer ist, je größer $\beta$ ist. $\beta$ ist also

\begin{tabular}{cllll}
\hline & \multicolumn{2}{c}{$z_{0}=5,55 \mathrm{~cm}$} & \multicolumn{2}{c}{$z_{0}=55,5 \mathrm{~cm}$} \\
$B$ & \multicolumn{3}{c}{$b / 2 z_{0}$} & \multicolumn{3}{c}{$\beta$} & $b / 2 z_{0}$ \\
\hline $10 \mathrm{G}$ & 1 & 0,70 & 10 & 0,42 \\
$160 \mathrm{G}$ & 8,15 & 0,43 & 81,5 & 0,15 \\
$1 \mathrm{kG}$ & 2,91 & 0,62 & 29,1 & 0,24 \\
$30 \mathrm{kG}$ & 1 & 0,72 & 10 & 0,38 \\
\hline
\end{tabular}

Tab. 3. Verhältnis $b / 2 z_{0}$ der Achtelwertsbreiten der Stromdichten in rotierenden und nicht rotierenden Plasmen.

ein $\mathrm{Ma} ß$ für die relative Rolle der Rotation des Plasmas: Für $\beta \gg 1$ ist die Rotation der dominierende Mechanismus für die Konzentration des 

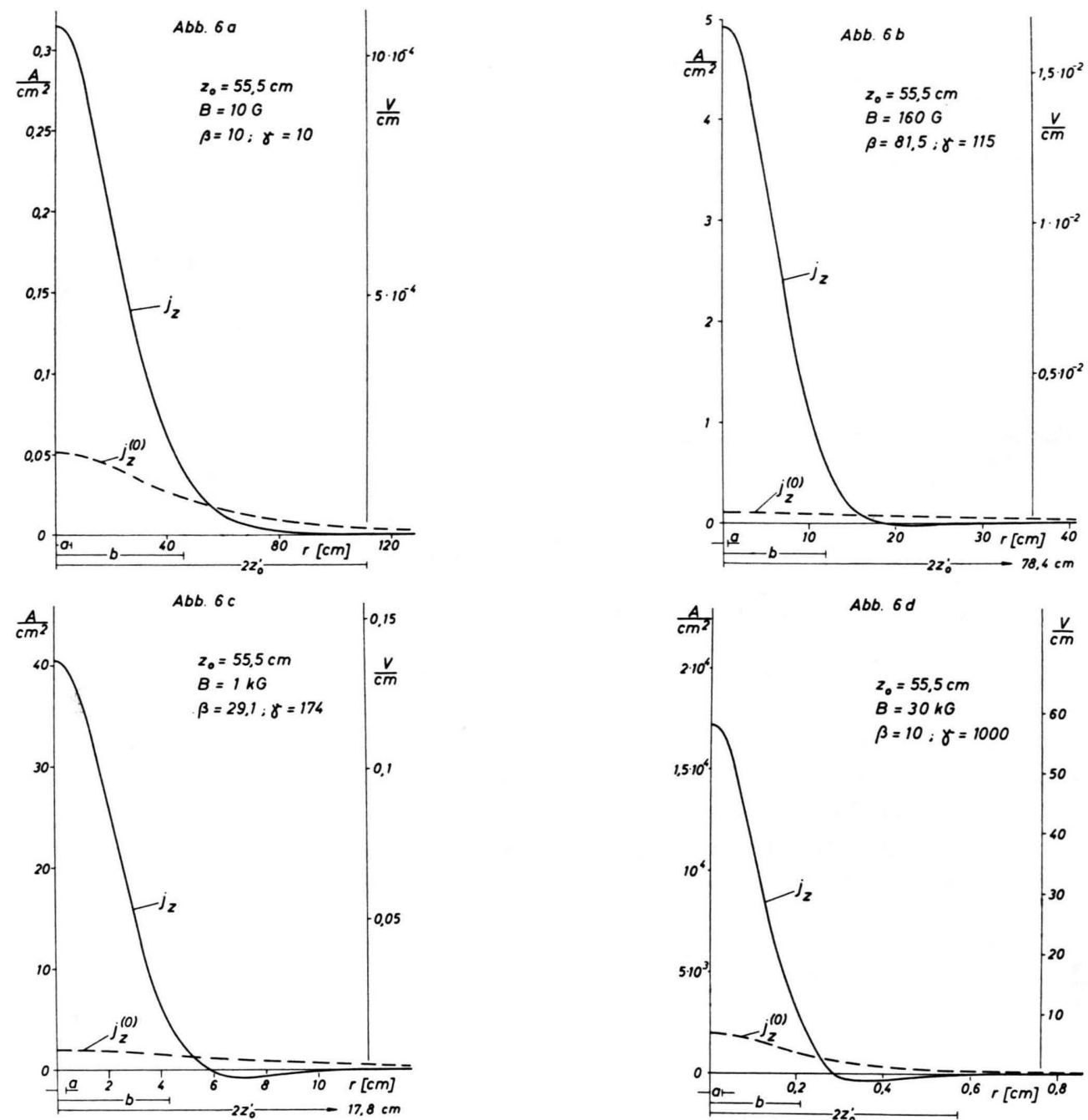

Abb. 6 a bis $6 \mathrm{~d}$. Stromdichten $j_{z}$ im rotierenden und $j_{z}^{(0)}$ im nicht rotierenden Plasma in der Mittelebene eines zwischen Punktelektroden brennenden Bogens. Elektrodenabstand $2 z_{0}=111 \mathrm{~cm}$. Plasmaparameter, Magnetfelder und Bogenstrom wie in Abb. 5 a bis $5 \mathrm{~d}$.

Bogenstromes auf die Achsenumgebung. Für $\beta \approx 1$ unterscheiden sich Achsenwerte und Achtelwertsbreiten der Stromdichten nicht um Größenordnungen. Nun ist $\beta$ zu $z_{0}$ proportional. Das bedeutet, daß die Rotation bei sonst festgehaltenen Parametern eine um so größere Rolle spielt, je größer der Elektrodenabstand ist. Daß das zu erwarten ist, wurde in Abschnitt 2 bereits ausgeführt. Entsprechend dem hier zugrundeliegenden Bogenmodell befinden sich die Elektroden auf festen Wänden, an denen $v_{\varphi}$ verschwindet. Die bremsende Wirkung dieser Wände macht sich natürlich um so weniger bemerkbar, je größer ihr Abstand ist. Besonders soll darauf hingewiesen werden, da $\beta$ die Breite $z_{0}^{\prime}=\sqrt{\sigma^{\prime} / \sigma} z_{0}$ zu $z_{0}$ proportional ist. Die Breite $b$ nimmt zwar auch mit $z_{0} \mathrm{zu}$, aber schwächer als linear, wie aus Tab. 3 hervorgeht. Weiterhin war oben gezeigt worden, daß $\beta$ für $\omega_{\mathrm{e}} \tau_{\mathrm{e}}=1$ sein Maximum hat. Für $\omega_{\mathrm{e}} \tau_{\mathrm{e}}=1$ ist also die Abweichung vom nicht rotierenden Plasma am größten, also dann, wenn der Hall-Strom noch keine sehr bedeutende Rolle spielt. Für $\omega_{\mathrm{e}}^{2} \tau_{\mathrm{e}^{2}} \ll 1$ ist der Hall-Strom praktisch unwirksam; die Stromdichteverteilung im nicht rotierenden Plasma unterscheidet sich dann nicht von der im Fall $B=0$. Die für $B=10 \mathrm{G}$ gefundenen Abweichungen zwischen $j_{z}$ und $j_{z}^{(0)}$ sind deshalb ausschließlich auf die Rotation des Plasmas zurückzuführen. 
Tab. 4 zeigt das Verhältnis der Achtelswertbreite $b$ der Stromdichte zu der für reibend rotierende Plasmen charakteristischen Länge $a$. Dieses Verhältnis wächst offenbar ebenfalls monoton und schwächer als linear mit $\beta$ an.

\begin{tabular}{cllllr}
\hline & & \multicolumn{2}{c}{$z_{0}=5,55 \mathrm{~cm}$} & \multicolumn{2}{c}{$z_{0}=55,5 \mathrm{~cm}$} \\
$B$ & & $\beta$ & $b / a$ & & $b / a$ \\
\hline $10 \mathrm{G}$ & 5,55 & 1 & 1,4 & 10 & 8,3 \\
$160 \mathrm{G}$ & 0,475 & 8,15 & 7,1 & 81,5 & 25,0 \\
$1 \mathrm{KG}$ & 0,317 & 2,91 & 3,5 & 29,1 & 13,5 \\
$30 \mathrm{KG}$ & 0,029 & 1 & 1,4 & 10 & 7,5 \\
\hline
\end{tabular}

Tab. 4. Verhältnis $b / a$ der Breite der Stromdichteverteilung zur charakteristischen Länge $a$.

Weiterhin ist etwas zur Rolle des Parameters $\gamma$ zu sagen. Für $B=10 \mathrm{G}$ und $B=30 \mathrm{kG}$ hat $\beta$ den gleichen Wert, aber für $B=10 \mathrm{G}$ ist $\gamma=\beta$, für $B=30 \mathrm{kG}$ jedoch $\gamma=100 \beta$. Die in den Tab. 2, 3 und 4 aufgeführten Verhältnisse unterscheiden sich aber für diese beiden Werte von $B$ kaum. Die Abb. 5 a bis $6 \mathrm{~d}$ und die Tab. 2 bis 4 liefern somit die Aussage, da $\beta$ der für die Stromdichteverteilung maßgebende Parameter ist. Diese Aussage läßt sich allgemein aus Gl. (18) herleiten. Das wird im zweiten Teil der Arbeit durchgeführt. Der erste Teil beschränkt sich auf eine weitere Diskussion der Re-

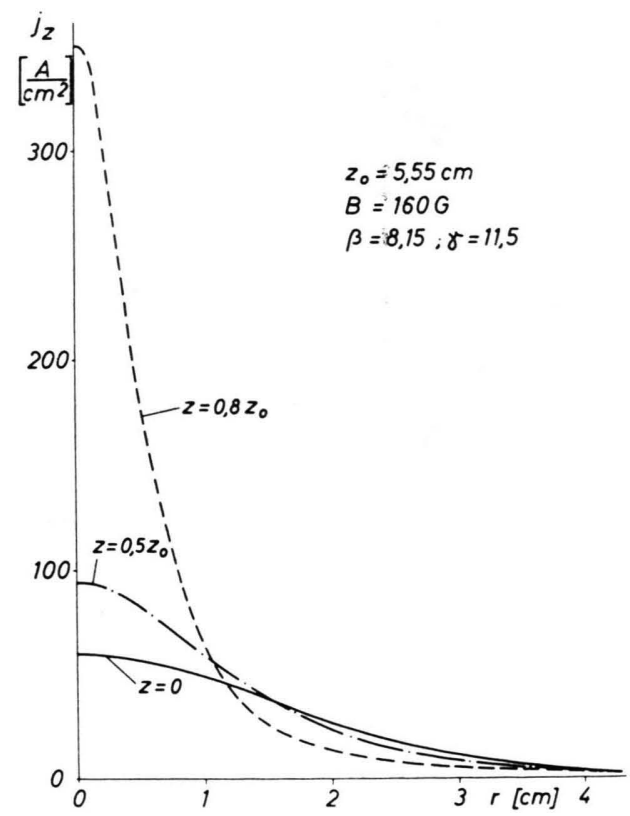

Abb. 7. Stromdichte $j_{z}$ im rotierenden Plasma in den Ebenen $\mathrm{z}=0$ (Mittelebene), $z= \pm 0,5 z_{0}$ und $z= \pm 0,8 z_{0}$. Elektrodenabstand $2 z_{0}=11,1 \mathrm{~cm} . \mathrm{B}=160 \mathrm{G}$. Plasmaparameter und Gesamtstrom wie bisher. sultate für die hier gewählten charakteristischen Parameter. Bisher wurde nur der Verlauf von $j_{z}(r)$ in der Mittelebene $z=0$ des Bogens gezeigt. Ein Beispiel für die Zunahme der Stromdichte zu den Elektroden hin gibt Abb. 7. Sie zeigt für $B=160 \mathrm{G}$ $\left(\omega_{\mathrm{e}} \tau_{\mathrm{e}}=1\right.$, Einfluß der Rotation am ausgeprägtesten) und $z_{0}=5,55 \mathrm{~cm}$ die Stromdichte $j_{z}(r)$ in der Ebene $z=0$ (also noch einmal die gleiche Kurve wie in Abb. 5b), sowie in den Ebenen $z=0,5 z_{0}$ und $z=0,8 z_{0}$. Der Verlauf der Stromlinien für

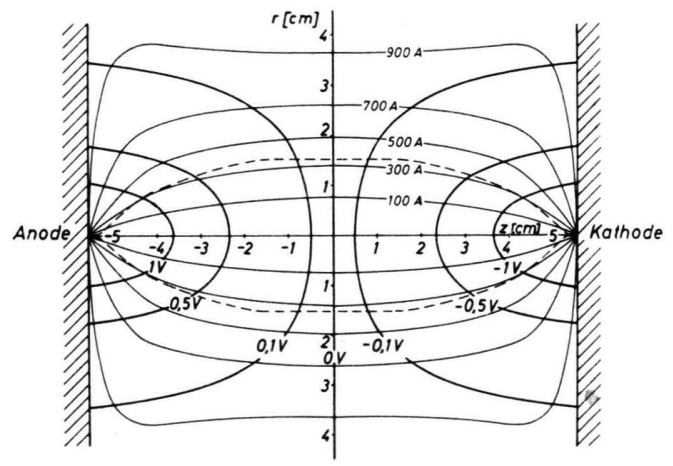

Abb. 8. Stromlinien und Äquipotentiallinien im rotierenden Plasma für einen zwischen Punktelektroden brennenden Bogen. Bogenstrom, Magnetfeld und Plasmaparameter wie in Abb. 7. Die gestrichelte Kurve bezeichnet den Ort, an $\operatorname{dem} \partial v_{\varphi} / \partial r=0$ ist.

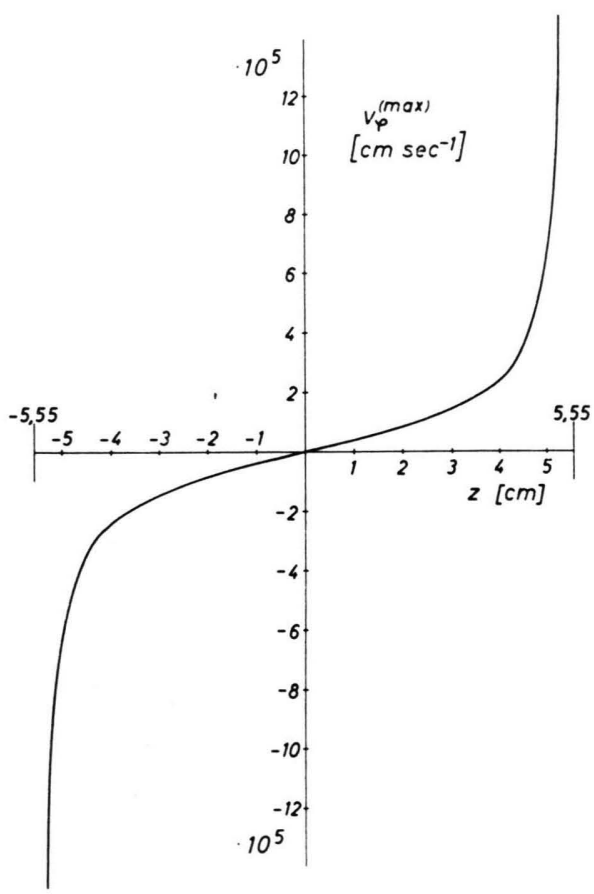

Abb. 9. Die Maximalwerte von $v_{\varphi}(r)$ als Funktion von $z$ für den in Abb. 8 dargestellten Bogen. 
$B=160 \mathrm{G}$ und $z_{0}=5,55 \mathrm{~cm}$ ist in Abb. 8 dargestellt. In dieser Abb. sind ferner einige Äquipotentiallinien eingezeichnet, sowie die Kurve, auf der $v_{\varphi}$ sein Maximum hat. Es sei noch einmal daran erinnert, daß $V, E_{\mathrm{r}}$ und $v_{\varphi}$ wegen der Symmetrieeigenschaften für $z=0$ verschwinden und auf den Elektroden ebenso wie $E_{z}$, bzw. $j_{z}$ divergieren. Die Maximalwerte von $v_{\varphi}$ sind in Abb. 9 aufgetragen.

Weiterhin soll an Beispielen gezeigt werden, wie weit die Radialkomponente $E_{\mathrm{r}}$ des elektrischen Feldes durch $v_{\varphi} B$ in den bisher behandelten Fällen
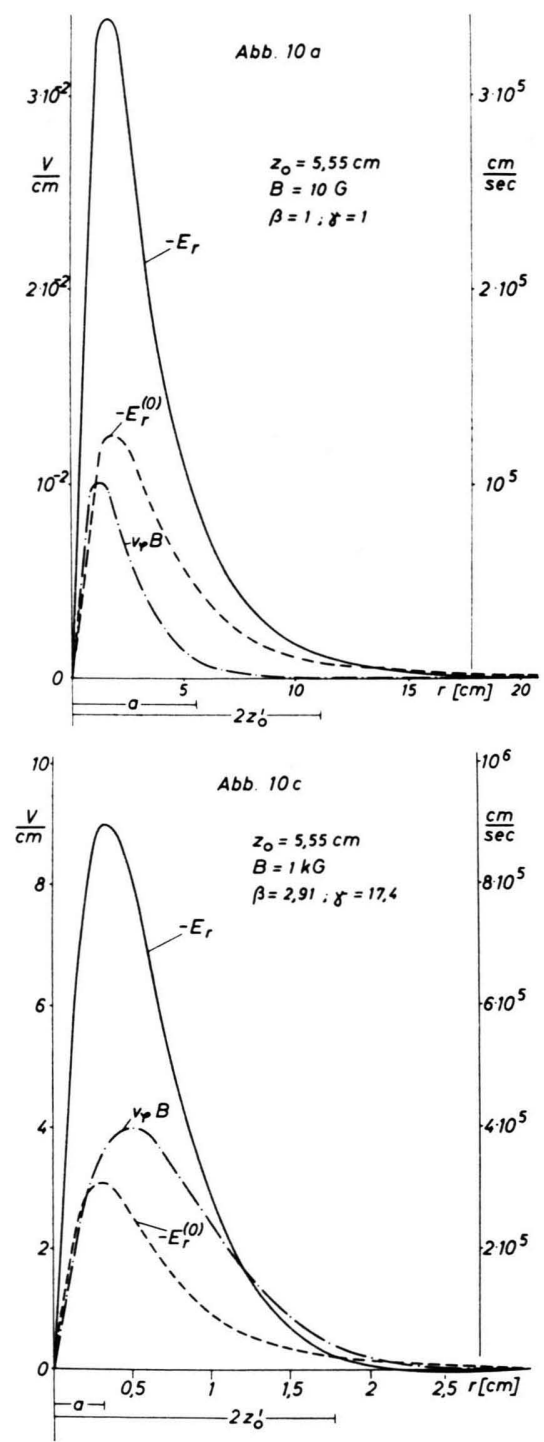

kompensiert wird. Dazu wurden $-E_{\mathrm{r}}$ und $v_{\varphi} B$ als Funktion von $r$ in der Ebene $z=0,5 z_{0}$ aufgetragen. Die Abb. 10a bis 10d entsprechen den in den Abb. 5a bis 5d zugrunde gelegten Parametern; ebenso gehören die Abb. 11 a bis $11 \mathrm{~d}$ zu den Abb. 6 a bis 6 d. Die Skala am rechten Rand dieser Abb. gibt $v_{\varphi}$ selbst an. Ferner wurde auch der Verlauf von $-E_{\mathrm{r}}^{(0)}$, der radialen elektrischen Feldstärke für nicht rotierendes Plasma aufgetragen. Da

$$
j_{\mathrm{r}}=\sigma^{\prime} E_{\mathrm{r}}+\sigma^{\prime} v_{\varphi} B
$$
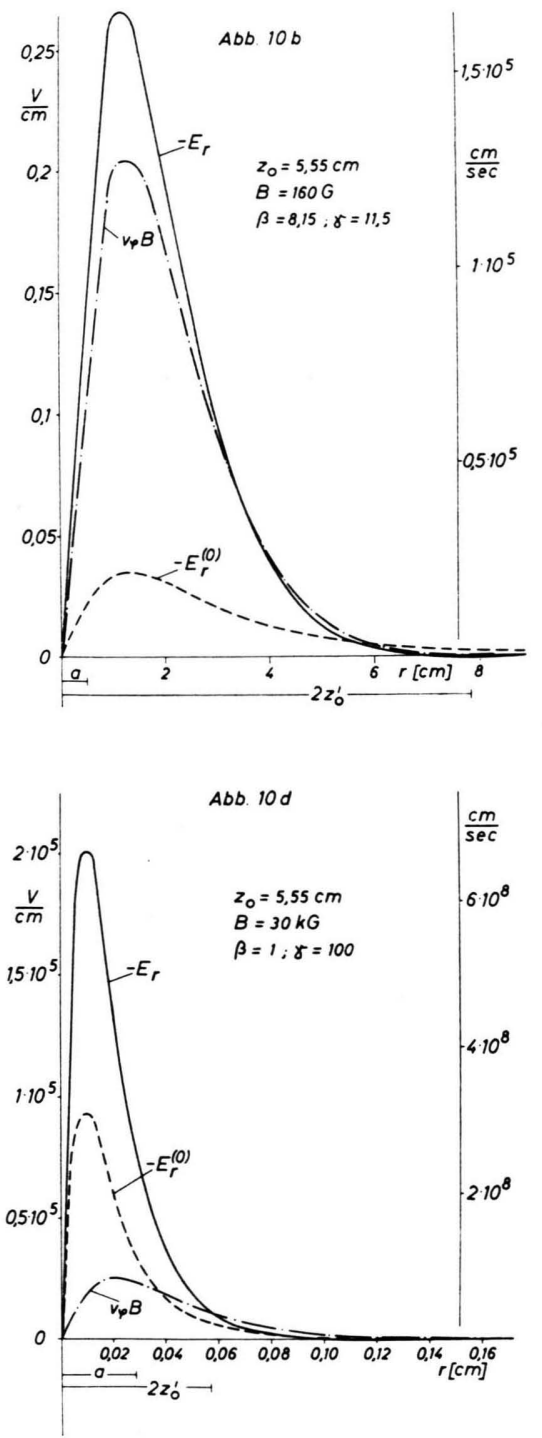

Abb. $10 \mathrm{a}$ bis $10 \mathrm{~d}$. $-E_{\mathrm{r}}$ und $v_{\varphi} B$ im rotierenden und $-E_{\mathrm{r}}^{(0)}$ im nicht rotierenden Plasma in der Ebene $z=0,5 z_{0}$. Punktelektroden im Abstand $2 z_{0}=11,1 \mathrm{~cm}$. Bogenstrom, Magnetfelder und Plasmaparameter wie bisher. Die Skala am rechten Rand gibt $v_{\varphi}$ an. 

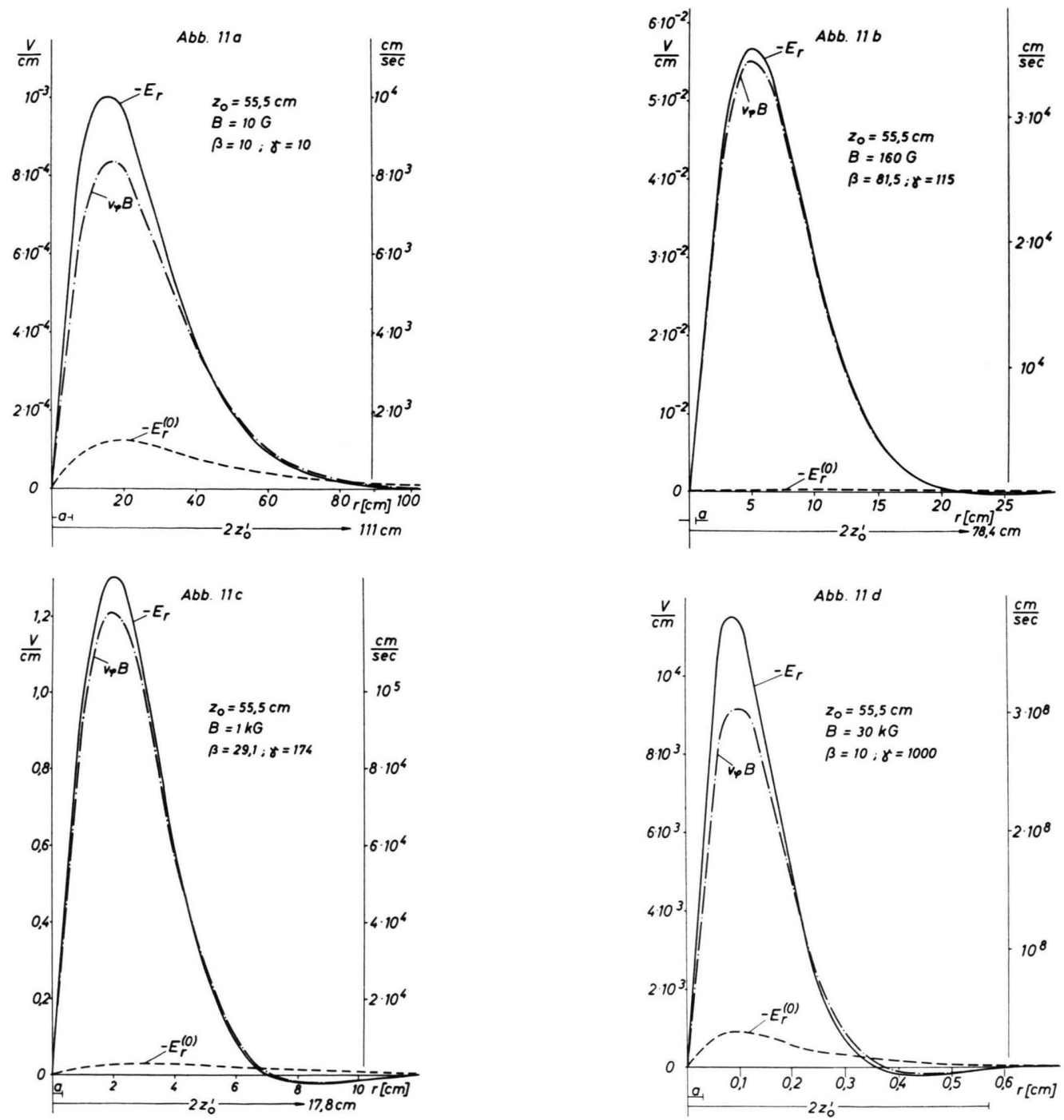

Abb. $11 \mathrm{a}$ bis $11 \mathrm{~d}$. $-E_{\mathrm{r}}$ und $v_{\phi} B$ im rotierenden und $-E_{\mathrm{r}}^{(0)} \mathrm{im}$ nicht rotierenden Plasma in der Ebene $z=0,5 \mathrm{z}_{0}$. Punktelektroden im Abstand $2 z_{0}=111 \mathrm{~cm}$. Bogenstrom, Magnetfelder und Plasmaparameter wie bisher.

ist, gestatten die Abb. 10 und 11 auch einen direkten Vergleich der Radialkomponenten der Stromdichte: Für nicht rotierendes Plasma ist $j_{\mathrm{r}} \mathrm{zu} E_{\mathrm{r}}$ proportional, für ein rotierendes Plasma proportional zur Differenz von $E_{\mathrm{r}}$ und $v_{\varphi} B$.

In den Abb. 10 und 11 fällt auf, daß $\left|E_{\mathrm{r}}\right|$ nicht überall größer als $\left|v_{\varphi} B\right|$ ist. Für ein reibungsfreies Plasma würde $E_{\mathrm{r}}=-v_{\varphi}^{*} B$ gelten und man hätte erwartet, daß die innere Reibung dazu führt, daß $\left|v_{\varphi}\right|$ hinter $\left|v_{\varphi}^{*}\right|$ zurückbleibt. Ein solches „,normales" Verhalten der Rotationsgeschwindigkeit liegt nach den Abb. 10 und 11 auch durchweg in der Achsenumgebung vor. Für größere Radien übertrifft $\left|v_{\varphi} B\right|$ jedoch meistens $\left|E_{\mathrm{r}}\right|$, so daß $j_{\mathrm{r}}$ dort sein Vorzeichen umkehrt. Daß dieses Ergebnis durch eine Ungenauigkeit in der numerischen Auswertung der Integration über $k$ zustande kommt, läßt sich ausschließen, denn es läßt sich zeigen, daß der Integrand von $\partial \hat{V} / \partial \zeta$ für große $k$ langsamer als die Integranden von $\hat{V}, \partial \hat{V} / \partial \varrho$ und $\hat{w}$ mit $k$ abnimmt. Die oben beschriebenen Kontrollen für die Konvergenz von $\partial \hat{V} / \partial \zeta \sim j_{z}$ garantieren deshalb auch für hinreichend genaue Berechnung von $E_{\mathrm{r}}$ und $v_{\varphi}$. Das ,, anomale" Verhalten der Rotationsgeschwindigkeit, d.h. $\left|v_{\varphi}\right|>\left|E_{\mathrm{r}} / B\right|$ ist somit ein echter Effekt, der sich auch physikalisch 
interpretieren läßt: Die Rotationsgeschwindigkeit an irgendeiner Stelle des Plasmas wird ja nicht nur durch den ,,lokalen Bedarf an Kompensation“ bestimmt, d.h. durch die Tendenz, den Zustand mit $j_{\mathrm{r}}=0$ anzustreben; sie ist vielmehr durch die innere Reibung auch mit der Rotationsbewegung der gesamten Umgebung gekoppelt. Deswegen kann das Plasma durchaus stellenweise schneller rotieren, als dem ,lokalen Bedarf“" entspricht. Man kann benachbarte Bereiche des Plasmas mit zwei Elektromotoren vergleichen, die gleichen Ohm'schen Widerstand haben, von Generatoren verschiedener Spannung angetrieben werden, und die mechanisch - etwa durch einen Transmissionsriemen - miteinander gekoppelt sind. Die Drehzahl beider Motoren wird dann nicht nur durch die jeweiligen Spannungen, sondern auch durch die Stärke der Kopplung bestimmt, so daß die Rotation eines Motors eine EMK liefern kann, die die Generatorspannung übertrifft. Dieser Vergleich hinkt allerdings insofern, als die Trennung zwischen Generator und Motor für ein Plasma nicht zulässig ist: Im rotierenden Plasma kann nach den Abb. 10 und 11 nicht nur $\left|v_{\varphi} B\right|>\left|E_{\mathrm{r}}\right|$ werden, sondern es kann auch $E_{\mathrm{r}}$ sein Vorzeichen wechseln und somit ,,anomal" werden.

Der hier diskutierte Effekt erklärt natürlich auch das bereits erwähnte ,,anomale“ Verhalten von $j_{z}$ für große Radien, das in den Abb. 5 und 6 zu erkennen ist. Es gibt also offensichtlich in manchen Fällen außer den ,,normalen“ Stromlinien, die von der Anode ausgehen und auf der Kathode enden,

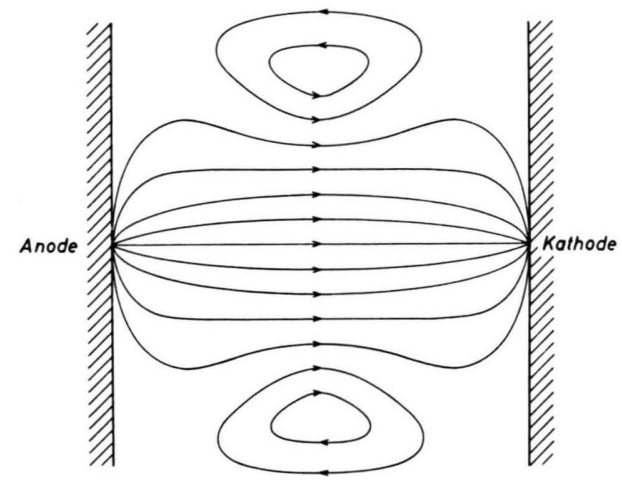

Abb. 12. Schematische Darstellung eines Lichtbogens mit ,,normalen" und ,,anomalen" Stromlinien. "Anomale“ Stromlinien treten auf, wenn $\left|v_{\varphi}\right|>\left|E_{\mathrm{r}} / B\right|$ ist. Dieser Effekt wird von der inneren Reibung des Plasmas hervorgerufen. Der hier gezeigte Stromlinienverlauf stimmt qualitativ mit Verhältnissen überein, wie sie z. B. in den Abb. $6 \mathrm{~d}$ und $11 \mathrm{~d}$ gezeigt sind. auch noch ,,anomale“ Stromlinien, die innerhalb des Plasmas in sich geschlossen sind. Abb. 12 zeigt schematisch, wie man sich ein solches Stromlinienbild anhand der hier diskutierten Ergebnisse vorzustellen hat. Die Berechnung solcher Stromlinien ist prinzipiell natürlich möglich, aber sehr aufwendig, da wegen der kleinen Beträge der anomalen Stromdichten, Feldstärken und Geschwindigkeiten eine sehr hohe Rechengenauigkeit erforderlich ist.

\section{Zusammenfassung}

Wird einem Lichtbogen ein äußeres axiales Magnetfeld überlagert, so beeinflußt dieses ganz entscheidend die radiale Verteilung der Stromdichte, und zwar auf Grund zweier Effekte: Eine Radialkomponente der Stromdichte führt zu einer Rotation des Plasmas mit der Geschwindigkeit $v_{\varphi}$ und einem azimutalen Hall-Strom $j_{\varphi}^{(\mathrm{H})}$. Diese liefern elektromotorische Kräfte, die - in Übereinstimmung mit der Lenz'schen Regel - der radialen Stromdichte entgegenwirken. Das Zusammenspiel beider Effekte wird einerseits durch die Geometrie des Bogens bestimmt, andererseits durch den HallParameter $\omega_{\mathrm{e}} \tau_{\mathrm{e}}$, das Produkt aus Gyrofrequenz und reziproker Stoßfrequenz der Elektronen, sowie durch die Viskosität des Plasmas, die praktisch mit der des Ionengases identisch ist.

In einem reibungsfreien Plasma kann kein stationärer Strom senkrecht zum äußeren Magnetfeld fließen; der Bogenstrom fließt also parallel zu den Feldlinien dieses Feldes, falls die Anordnung der Elektroden das zuläßt. Das trifft z. B. für auf der Achse gelegene Punktelektroden zu; dieser Fall wird im ersten Teil der Arbeit stets betrachtet. Für ein reibendes Plasma hängt die Verbreiterung der Stromdichteverteilung dann vom Elektrodenabstand und von einer charakteristischen Länge a ab, in die die Leitfähigkeit, der Hall-Parameter und die Viskosität des Plasmas, bzw. Dichte, Temperatur und magnetische Feldstärke eingehen. Für ein Bogenmodell - homogenes Plasma in einfacher Geometrie - lassen sich die die stationäre Stromverteilung bestimmenden Gleichungen $-\operatorname{rot} \boldsymbol{E}=0$, $\operatorname{div} \boldsymbol{j}=0$, Bewegungsgleichung (Navier-Stokes-Gl.) und verallgemeinertes Ohmsches Gesetz - exakt lösen.

Die Lösungen werden mit denen für ein nicht rotierendes Plasma verglichen, in dem das Magnetfeld ebenso wie in einem festen Metall nur über den 
Hall-Effekt auf die Elektronen einwirkt. Der Vergleich zeigt, daß der Bogenstrom im rotierenden Plasma stets wesentlich stärker auf die Achsenumgebung konzentriert ist als im nicht rotierenden Plasma. Physikalisch bedeutet das, daß der Stromfluß in einem Lichtbogen mit äußerem Magnetfeld nicht nur durch das Verhalten der Elektronen, sondern ganz wesentlich auch durch das Verhalten der Ionen bestimmt wird. Als Maß für die Bedeutung der Rotationsbewegung läßt sich ein Parameter $\beta$ angeben, der vom Elektrodenabstand, von der magnetischen Feldstärke und von den Plasmaeigenschaften abhängt.

Die vorgelegten Resultate zeigen ferner, daß die Radialkomponente der elektrischen Feldstärke durch $v_{\varphi} B$ überkompensiert werden kann. Das hat dann zur Folge, daß im Plasma außer dem Bogenstrom ein ,,sekundärer" Strom mit in sich geschlossenen Stromlinien hervorgerufen wird.
Im zweiten Teil der Arbeit wird gezeigt werden, daß der Parameter $\beta$ nicht nur für die bisher diskutierten Beispiele, sondern ganz allgemein die für die Stromdichteverteilung maßgebende Größe ist. Das gilt auch für nicht punktförmige Elektroden, für die dann ebenfalls Resultate vorgelegt werden. Weiterhin werden Betrachtungen darüber angestellt, inwieweit sich die mit Hilfe des Bogenmodells gewonnenen Resultate auf reale Lichtbögen anwenden lassen. Unter Beachtung der dabei gewonnenen Kriterien wird darauf ein Vergleich mit experimentellen Ergebnissen vorgenommen.

Herrn Dr. H. WulfF danke ich für zahlreiche klärende Diskussionen, Herrn H. GoRenflo für die Programmierung und sorgfältige Durchführung der numerischen Rechnungen. Diese Arbeit wurde im Rahmen des Vertrages zwischen dem Institut für Plasmaphysik und der Europäischen Atomgemeinschaft über die Zusammenarbeit auf dem Gebiet der Plasmaphysik durchgeführt. 